\title{
Information continuity across the response selection bottleneck: Early parallel Task 2 response activation contributes to overt Task 2 performance
}

\author{
Sandra J. Thomson • Scott Watter
}

Published online: 17 April 2013

(C) Psychonomic Society, Inc. 2013

\begin{abstract}
Several studies of dual-task performance have demonstrated Task 2 to Task 1 response priming (backward compatibility effects), indicating some degree of parallel response computation for concurrent tasks and suggesting that the well-established response selection bottleneck (RSB) model may be incomplete. However, the RSB might be considered to remain informationally intact if this early parallel Task 2 response information does not persist across the attentional shift between tasks to contribute to overt Task 2 performance. We used an adapted psychological refractory period paradigm with an additional early transient Task 2 stimulus to examine whether response information generated for Task 2 in parallel with overt Task 1 response selection could persist across the bottleneck to influence eventual overt Task 2 performance. After controlling for potential indirect effects of Task 1 processing stage variability propagating onto Task 2 reaction time via locus of slack effects, we observed reliable and consistent effects of early Task 2 response information facilitating Task 2 reaction times. These effects were observed only when the responses to both tasks of the dual-task pair were compatible, under both univalent and bivalent response mappings across tasks. These findings may represent evidence of a variably sensitive response gating or suppression mechanism in dual-task performance and support the idea that backward response compatibility effects represent transient informational
\end{abstract}

\section{S. J. Thomson $(\triangle) \cdot$ S. Watter}

Department of Psychology, Neuroscience \& Behaviour, McMaster

University, 1280 Main Street West,

Hamilton, Ontario L8S 4K1 Canada

e-mail: thomsosj@mcmaster.ca

S. Watter

e-mail: watter@mcmaster.ca influences on central response codes, rather than later postbottleneck response execution processes.

Keywords Dual-task performance $\cdot$ Psychological refractory period $\cdot$ Response selection bottleneck $\cdot$ Backward compatibility effects $\cdot$ Response activation

People commonly experience difficulty in performing multiple tasks concurrently. This limitation in human performance has been studied extensively using the psychological refractory period (PRP) paradigm. In these studies, participants are presented with two stimuli and are asked to perform a given task for each in succession. The stimulus onset asynchrony (SOA) is varied, and the typical finding is that while the first task is unaffected by SOA, the time required to perform the second task increases as the SOA decreases and the two tasks overlap more in time. This PRP effect is taken as empirical evidence of a fundamental limitation in human central information processing.

The delay in performance for the second of two tasks can be explained by the RSB theory (Pashler, 1984, 1994; Pashler \& Johnston, 1998) and the related locus of slack logic. According to RSB theory, processing can proceed in parallel for two tasks until they reach a central stage of processing that can be performed only for one task at a time. If processing of the second task reaches the bottleneck stage while this stage is still occupied by Task 1 , Task 2 processing is temporarily suspended until Task 1 completes the bottleneck process and Task 2 can gain access to it. The bottleneck is presumed to be in the stage of response selection, where the response to a task is selected according to task set mapping rules. Pre- and postbottleneck processing stages of the two tasks can proceed in parallel; only the bottleneck 
A

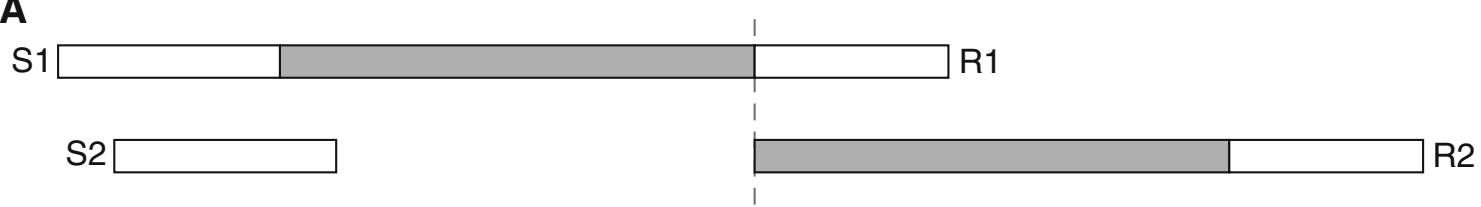

B
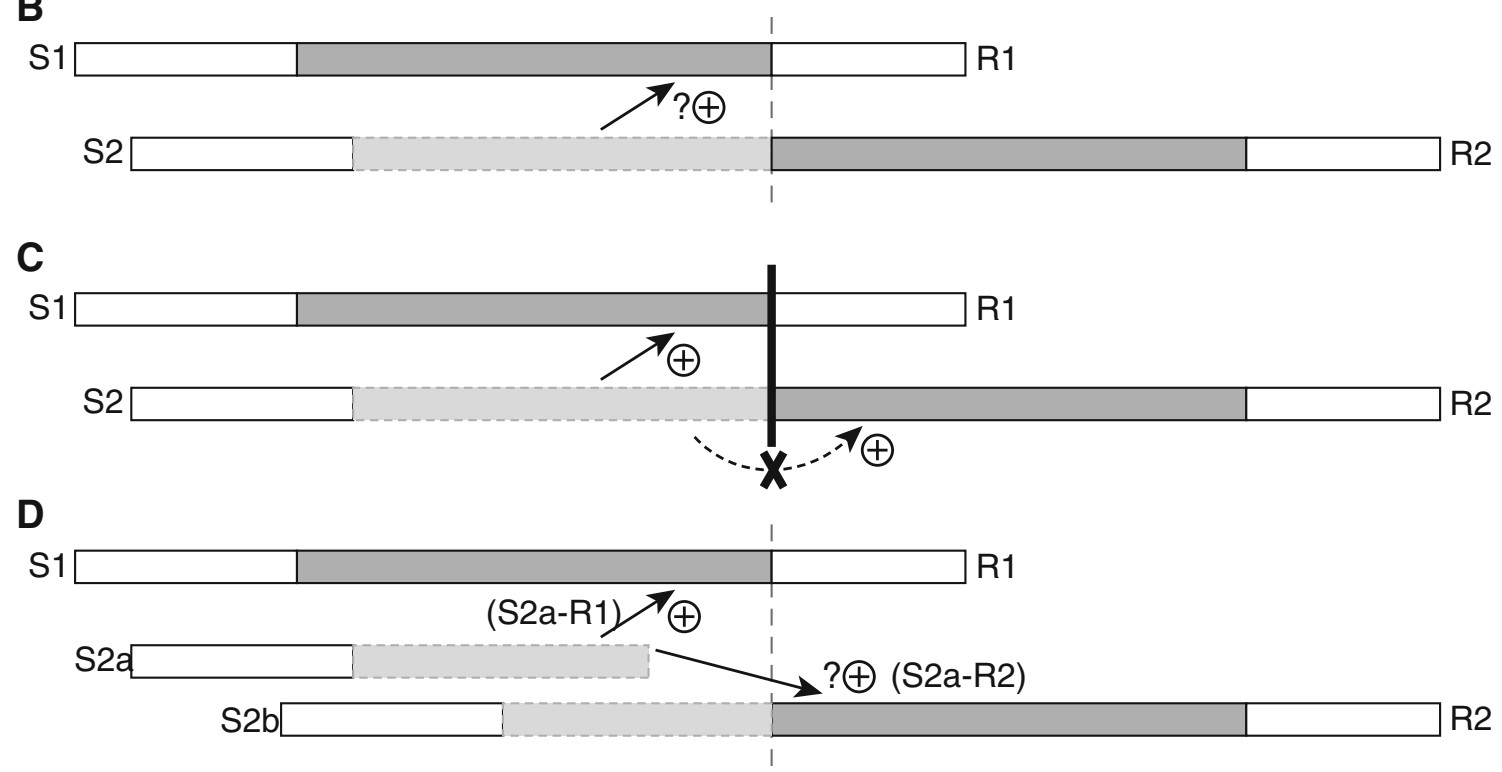

Fig. 1 Possible variations in dual-task processing at short SOAs. Dark gray shading indicates the response selection stage; light gray shading indicates response activation in Task 2 operating in parallel with Task 1 response selection. a The traditional response selection bottleneck model. b The Task 2 to Task 1 response priming effect demonstrated by Hommel (1998), Watter and Logan (2006), and others (“+” indicates response compatibility). c Illustration of the potential for suppression of response activation following the completion of Task 1 response selection to preserve a strict informational bottleneck in response selection. In this

stage in each task cannot overlap. This basic model is demonstrated in Fig. 1a.

\section{Evidence for parallel response selection}

While the RSB theory has proven to be quite robust and has been able to explain a wide range of results in dual-task studies, it has been challenged by several studies demonstrating an influence of Task 2 response selection processing on Task 1 performance. For instance, Hommel (1998) found that participants were faster to make a manual left/right response in Task 1 if Task 2 required a related vocal response (saying "left" or "right"). The presence of these backward compatibility effects implies that the response to Task 2 was activated before response selection for Task 1 was complete. Several other studies of backward cross talk in more traditional PRP paradigms have provided converging evidence that response information for Task 2 is activated early enough to influence situation, early parallel Task 2 response information can prime Task 1 response selection but does not influence overt Task 2 response selection. d The design of the present experiments, to test whether early parallel Task 2 response information can influence the eventual Task 2 response. Presenting a brief transient Task 2 stimulus (S2a) prior to the main Task 2 stimulus (S2b) allows the observation of potential response compatibility effects of early parallel S2a-related response information on the overt Task 1 response (S2a-R1 compatibility) and the overt Task 2 response ( $\mathrm{S} 2 \mathrm{a}-\mathrm{R} 2$ compatibility)

Task 1 responding (Miller, 2006; Miller \& Alderton, 2006; Thomson, Watter, \& Finkelshtein, 2010; Watter \& Logan, 2006). As is demonstrated in Fig. 1b, manual responses to Task 1 have been shown to be faster if the response key required for Task 2 is identical to that required for Task 1 , relative to when the two responses are different (e.g., Thomson et al., 2010; Watter \& Logan, 2006). Moreover, this Task 2 to Task 1 response priming can occur even for Task 2 stimuli that have not previously been encountered, suggesting that Task 2 response information can be automatically activated from semantic category representations of the Task 2 stimulus, independently of specific stimulus-response relationships (Thomson et al., 2010). Such findings imply that at least some portion of Task 2 response selection is occurring in parallel with Task 1, which would seem to be incompatible with a strict RSB account of dual-task performance.

There are a number of accounts of parallel response selection mechanisms in dual tasks. According to Meyer and Kieras's (1997a, b) executive process interactive control 
(EPIC) architecture, response selection may proceed in parallel for two tasks but participants strategically defer their responses for the second task. In Logan and Gordon's (2001) executive control theory of visual attention (ECTVA), the two tasks may be processed in parallel, but PRP performance is improved when the tasks are performed serially because of reduced interference between tasks. In accounting for his Task 2 to Task 1 response priming data, and mindful of the substantial and persistent Task 2 reaction time (RT) costs even in the presence of parallel response activation, Hommel (1998) suggested a model of separate stimulus-response translation (or response activation) and subsequent response selection processes to replace the traditional unified stage of response selection. The first of these distinct processes can occur automatically and in parallel with processing in a separate task and results in the activation of a response. Afterward, controlled rule-governed processes guide the ultimate selection of the response for each task in a serial fashion. The serial nature of this later response selection stage results in delayed execution of the second task. This version of a bottleneck model does not include the computation of responses in its bottleneck stage. In considering Hommel's (1998) model, however, Watter and Logan (2006) questioned the nature of the later serial selection stage. Specifically, if the work of accruing response information from stimuli and activating a response for each task is completed in the initial parallel response activation stage, what work is then left for the serial response selection stage that supposedly is the source of the processing delay? In this situation, the serial response selection process takes on a very different character than typically described and tested in traditional locus of slack PRP studies.

\section{The fate of response information for Task 2 generated in parallel with Task 1}

Accumulating dual-task findings have argued for Task 2 response information being generated in parallel with Task 1 performance and prior to completion of Task 1 response selection. However, an alternative account is suggested by the persistence of large RT costs on Task 2 performance. It is possible that the parallel response activation for Task 2, reflected by Task 2 to Task 1 response priming effects observed by Hommel (1998), Watter and Logan (2006), Miller (2006), and others, may not actually involve any influence on eventual attended Task 2 performance. Instead, early response activation for Task 2 may occur entirely automatically and in parallel with attended Task 1 performance, but when participants' attention subsequently switches to Task 2, any response information that has been generated up to that point is disregarded and does not contribute to the actual performance of the second task. This possibility is illustrated in Fig. 1c. This account preserves the spirit of the RSB theory, since response selection for Task 2 must essentially start over once attention is focused on this task, and it can also explain the influence of Task 2 processing on Task 1 performance. Importantly, the phase of response selection that actually contributes to attended performance of Task 2 is functionally and informationally discrete from the response selection stage of Task 1.

The idea that any Task 2 response information derived in parallel with Task 1 response selection may not be used in the eventual selection of a response for Task 2 is not new. Computational models of dual-task performance such as ECTVA (Logan \& Gordon, 2001) employ response inhibition mechanisms between tasks in order to prevent response perseveration and interference from Task 1 processing. Inhibiting or resetting response counters after Task 1 response selection is complete would also result in the loss of any partial activation of response information for Task 2 generated in parallel with Task 1, so that this information has no influence on attended Task 2 performance. According to this view, the Task 2 to Task 1 priming studies mentioned previously do not directly address the discreteness assumption of the RSB theory. From this view, the critical test of the informational bottleneck idea is not backward response priming from Task 2 to Task 1 but, instead, priming from early unattended Task 2 response information to subsequent attended Task 2 performance. Does Task 2 response information generated automatically and in parallel with Task 1 response selection persist beyond the bottleneck and the attentional shift from Task 1 to Task 2, in order to influence actual Task 2 performance?

Schubert, Fischer, and Stelzel (2008) recently addressed this question. They used a PRP paradigm where participants discriminated between tones for Task 1 and judged the direction of a visually presented arrow for Task 2 . Importantly, the Task 2 stimulus was preceded by a subliminal prime arrow that was congruent, incongruent, or neutral with respect to the target arrow. Schubert et al. measured the effect of Task 2 prime congruency on Task 1 and Task 2 RTs (RT1 and RT2) and observed an underadditive prime congruency effect in RT2 that was significant at short stimulus onset asynchronies (SOAs).

The authors considered two alternative models of how response activation arising from the early Task 2 prime stimulus could affect Task 2 performance. One possibility is that response information generated for the Task 2 prime stimulus in parallel with attended Task 1 processing persists across the bottleneck to directly influence Task 2 response selection. They labeled this option the bypass model. Alternatively, according to the indirect influence model, response activation for the Task 2 prime stimulus has no direct access to attended response selection in Task 2. Instead, a mechanism mediated solely by cross talk between tasks mimics the effects of the bypass model. On response-compatible trials, 
cross talk from Task 2 to Task 1 leads to a shortening of Task 1 prebottleneck or bottleneck processing stages. The bottleneck stage of Task 2 can therefore begin earlier and shorten the overall duration of Task 2 by the same amount. Conversely, on incompatible trials, cross talk from Task 2 to Task 1 lengthens these Task 1 stages, and as a result, Task 2 processing is further delayed. In these situations, bottleneck or later processing stages of Task 2 are not influenced by response information from early Task 2 prime stimuli directly, but RT2 is shortened or lengthened due to propagated Task 1 effects via the locus of slack at short SOAs. In this view, the accumulated response information for Task 2 is reset or otherwise does not actually contribute to later Task 2 processing.

Schubert et al. (2008) found a congruence effect between the prime and target Task 2 stimuli only in situations where informational overlap existed between tasks, allowing for the possibility of response information cross talk from Task 2 to Task 1 and subsequent indirect propagation of Task 2 congruency effects onto RT2. In addition, the magnitude of the Task 2 congruency effects were observed to be equivalent in both RT1 and RT2, providing further support for the indirect mediation of Task 2 congruency effects. From these results, Schubert et al. concluded that early response activation for Task 2 does not bypass the RSB and does not persist to directly influence overt Task 2 performance.

\section{The present study}

The goal of the present study was to provide an additional test of the bypass model. The question of whether information can persist across the RSB is critical to the RSB theory specifically and to our understanding of dual-task processing in general. Evidence of parallel response selection alone is not sufficient to contradict the bottleneck model if the result of this processing does not contribute to secondary task performance. In fact, this resetting of accumulated response information would resolve the apparent paradox of backward compatibility effects in RT1 despite the persistence of substantial dual-task costs observed in RT2. However, demonstrations of information continuity across the bottleneck would be incompatible with central assumptions of the RSB theory. Given these important implications, we feel that it is worthwhile to investigate this issue further.

We employed a design that could independently assess response compatibility priming between tasks, as well as potential influences from early parallel response activation in Task 2 to later overt Task 2 performance. Our design involved a typical PRP paradigm with different tasks for Task 1 and Task 2. We used a stimulus substitution technique, similar to that in Schubert et al. (2008), where the initial stimulus for Task 2 (S2a) was replaced by a second, target Task 2 stimulus
(S2b) after a short 200-ms interval, with participants told to ignore the early transient $\mathrm{S} 2 \mathrm{a}$ and respond to the subsequent $\mathrm{S} 2 \mathrm{~b}$, presented for $1,000 \mathrm{~ms}$. This allowed us to assess the influence of any response information potentially generated for this initial transient S2a stimulus on both Task 1 processing (by examining backward response compatibility from Task 2 to Task 1), and eventual explicit Task 2 performance. Our transient stimulus was displayed substantially longer than that of Schubert et al. (only $34 \mathrm{~ms}$ ), which may provide a better opportunity for sufficiently strong response activation to persist across the bottleneck (although for a demonstration of subliminal Task 2 primes generating compatibility effects in Task 1 and Task 2, see Fischer, Kiesel, Kunde, \& Schubert, 2011).

Our general design is shown in Fig. 1d. If response information is generated for the transient $\mathrm{S} 2 \mathrm{a}$ in parallel with response selection processes in Task 1, we expect to find evidence of Task 2 to Task 1 response compatibility effects from the S2a stimulus (S2a-R1 compatibility on RT1) similar to those demonstrated by Watter and Logan (2006), Miller (2006), Miller and Alderton (2006), and Thomson et al. (2010), even though S2a itself did not require a response. Additionally, we assessed Task 2 for the potential influence of S2a compatibility on explicit Task 2 performance (S2a-R2 compatibility on RT2), and we expect results similar to those of Schubert et al. (2008), where this effect was underadditive with SOA but still prominent even at short SOAs. We also assessed both Task 1 and Task 2 for expected effects of response compatibility between tasks (R1-R2 compatibility on both RT1 and RT2, not illustrated in Fig. 1d).

Critically, after observing S2a-R2 compatibility effects on Task 2, we conducted subsequent analyses on short SOA data to more conclusively assess whether this Task 2 effect was mediated by the indirect propagation of Task 2 to Task 1 prebottleneck or bottleneck cross talk effects onto RT2 (as suggested by Schubert et al., 2008) or whether, instead, response information generated from S2a could be observed to directly influence RT2 independently of any indirect propagation via Task 1. For this analysis, we calculated the difference between RT1 and RT2 on each correct trial and used this difference score as the dependent measure. For short-SOA data, subtracting the RTs for Task 1 from those of Task 2 should equate RT2 measures for any duration differences in Task 1 prebottleneck or bottleneck stages - in our present case, removing potential effects of priming of R1 from S2a response information that might propagate onto RT2. If this indirect propagation of Task 1 priming effects from $\mathrm{S} 2 \mathrm{a}$ is solely responsible for the $\mathrm{S} 2 \mathrm{a}-\mathrm{R} 2$ compatibility effect observed in the previous RT2 analysis, we should observe no S2a-related compatibility effects on these adjusted RT2 scores. If, however, a sufficient amount of response information generated from S2a in parallel with Task 1 processing persists across the bottleneck to influence overt Task 2 performance, we would expect to still find $\mathrm{S} 2$ a compatibility effects 
at short SOAs in the adjusted RT2 data. The latter case would suggest that S2a-related response information was able to bypass the bottleneck and directly influence overt Task 2 performance.

It is important to note that this type of analysis is relevant only at short SOAs, when processing in Task 2 is delayed by the bottleneck stage in Task 1. At longer SOAs, Task 2 is less affected by (or eventually, independent of) bottleneck processing delays from Task 1, and therefore, subtracting RT1 from RT2 becomes less meaningful. For this reason, this analysis was conducted only for our shortest two SOAs (0 and $100 \mathrm{~ms}$ ), where a strong PRP effect was observed, with a slope approaching -1 across compatibility conditions.

\section{Experiment 1}

Participants performed a PRP task. For Task 1, they were presented with a letter and were asked to indicate with a manual keypress response whether the letter was an $\mathrm{X}$ or a Z. Task 2 was a color discrimination task with two colors mapped to each response. Importantly, there were two Task 2 stimuli presented on every trial. The first, S2a, was presented briefly, and participants were instructed to ignore it. The second, S2b, replaced S2a after $200 \mathrm{~ms}$, and it was to the latter Task 2 stimulus that participants made their response. The analysis investigated whether a response was activated for S2a in parallel with Task 1 performance and whether this response information was able to bypass the bottleneck to contribute to subsequent Task 2 performance.

\section{Method}

\section{Participants}

Twenty undergraduate students enrolled in psychology courses at McMaster University (15 females) participated in the experiment for partial fulfillment of course credit. All had normal or corrected-to-normal vision, and all were righthanded.

\section{Apparatus and stimuli}

The experiment was conducted on a Dell Dimension 4600 computer using a ViewSonic Professional series P95f + monitor and Presentation ${ }^{\circledR}$ (v.13, www.neurobs.com) experimental software. The stimuli for Task 1 were the letters $\mathrm{X}$ and Z, and for Task 2 they were squares presented in red, green, blue, or yellow. The letters were always presented in white, and all stimuli were presented against a black background. Participants sat at a viewing distance of approximately $60 \mathrm{~cm}$, and from this distance, the square stimuli subtended $0.9^{\circ}$ of visual angle in height and width, and the letter stimuli subtended $1^{\circ}$ of visual angle in height and $0.8^{\circ}$ in width. Stimuli were presented centrally, with letter stimuli for Task 1 always presented above the colored square stimuli for Task 2, with a vertical separation between the nearest edges of Task 1 and Task 2 stimuli of approximately $0.4^{\circ}$.

\section{Procedure}

For the Task 1 stimulus (S1), participants performed a letter discrimination task, indicating whether the letter stimulus was an X or a Z. For Task 2, participants were instructed to ignore the first S2a "distractor" square and respond only to the second S2b "target" square, by pressing one key if the target square was either red or yellow or a different key if the square was either blue or green. Participants made separate responses to each task by pressing the " 1 " or " 2 " key on the number pad of a standard keyboard with the index or middle finger of the right hand, for each task in sequence. The response mapping for each task was counterbalanced across participants, and a card was attached to the bottom of the monitor to remind participants of the mapping for their condition. Task 1 and Task 2 were separated in time by an SOA of $0,100,300$ or $900 \mathrm{~ms}$, defined as the separation of stimulus onset for S1 and S2a, with S2b replacing S2a $200 \mathrm{~ms}$ following S2a presentation.

The sequence of a single trial is illustrated in Fig. 2. Every trial lasted 4,000 ms and began with a fixation display for $500 \mathrm{~ms}$. This display consisted of two rows of two dashes centered on the screen, separated laterally in each row by approximately $1.1^{\circ}$ of visual angle, flanking the locations where the stimuli for each task would appear. On zero SOA trials, this display was replaced by a display containing a letter (S1) and a colored square (S2a) for $200 \mathrm{~ms}$, after which S2a was replaced with the target colored square $\mathrm{S} 2 \mathrm{~b}$. S1 and S2b were presented together for $1,000 \mathrm{~ms}$, followed by a blank screen for 2,300 ms. On nonzero SOA trials, the fixation display was replaced with a display containing only S1 for the duration of the SOA, after which S2a was added for $200 \mathrm{~ms}$, before being replaced by S2b. S1 and S2b remained onscreen together for $1,000 \mathrm{~ms}$, followed by a blank screen for the remainder of the trial duration. Each stimulus and SOA duration were presented an equal number of times, with the constraint that each S2a distractor was always followed by either the other color requiring the same response (compatible) or a consistent one of the two potential incompatible colors, each with equal probability. For example, a blue S2a was followed by a green S2b (response compatible) $50 \%$ of the time, and the other $50 \%$ of the time, it was followed by a red $\mathrm{S} 2 \mathrm{~b}$ (response incompatible). It was never followed by the other incompatible (yellow) S2b. The design was balanced such that neither the specific S2b color nor the response compatibility could be reliably predicted from S2a, and the $\mathrm{S} 2 \mathrm{a}$ and S2b colors were never identical on a given trial. 


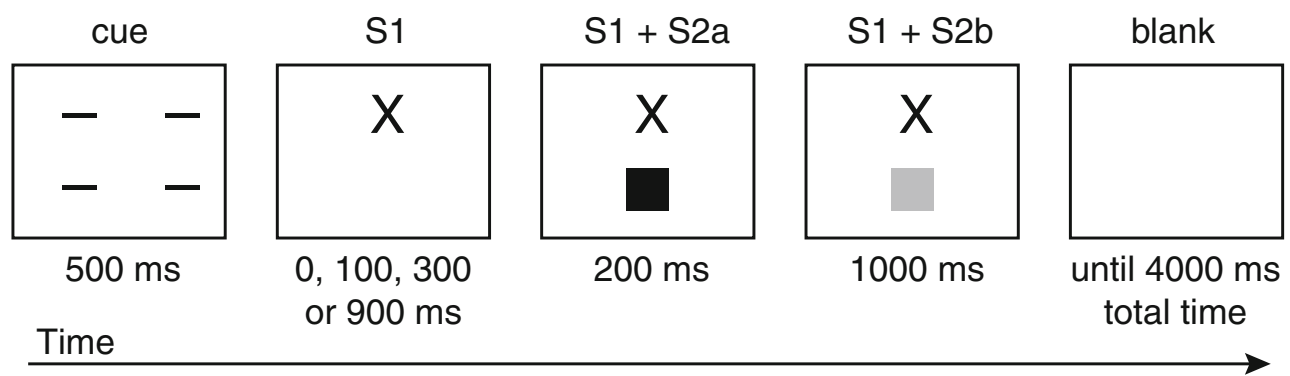

Fig. 2 The sequence of task elements within a single trial for Experiments 1 and 2. In Experiment 1, participants responded to Task 1 and then Task 2 with the index versus middle fingers of the same hand (bivalent mapping). In Experiment 2, participants responded with the index versus middle fingers of one hand for Task 1 and then with the

Participants were told that the experiment was a test of their concentration, to investigate how effectively they could complete simple tasks presented in quick succession. They were instructed that Task 1 was most important and that they should concentrate fully on this first task until its completion before attending to the colored square for Task 2. It was explicitly stated that they should not wait until the stimuli for both tasks were displayed before making their response to S1 but, instead, should be as quick and accurate as possible for each task in turn. Participants were informed of S2a but were told that this stimulus was irrelevant and were encouraged not to be distracted by it.

The experiment consisted of 512 trials, made up of eight iterations of the factorial combination of the two letter stimuli for Task 1, the four colors for S2a, the two potential colors that followed each as S2b (compatible or incompatible), and the four SOAs. Trials were presented in random order across 12 blocks, with 11 blocks of 43 trials and a final block of 39 trials. Participants were given the opportunity to rest before initiating the beginning of each block. Before the experimental trials began, participants completed a practice block of 32 trials that were not considered for analysis.

\section{Data analysis}

Data were analyzed from participants who completed Task 1 and Task 2 correctly on at least $70 \%$ of the trials. Trials with RTs less than 200 ms were excluded from analysis, as well as trials with RT1 greater than 1,500 ms or RT2 greater than 2,000 ms. Mean RTs for each condition were computed from the remaining trials where both Task 1 and Task 2 were correct. The initial analyses assessed the influence of response priming from both Task 2 stimuli ( $\mathrm{S} 2 \mathrm{a}$ and $\mathrm{S} 2 \mathrm{~b}$ ) on Task 1 performance, as well as response priming in Task 2 from both Task 1 and the distractor S2a. For Task 1, we evaluated the response compatibility between Task 1 and Task 2 responses (R1-R2 compatibility), as well as the compatibility between $\mathrm{R} 1$ and the theoretical response to index versus middle fingers of their other hand for Task 2 (univalent mapping). Task 1 required participants to respond to $S 1$ letter identity ("X" vs. "Z"). Task 2 required participants to respond to S2b color (red or yellow vs. blue or green) and ignore the initial transient S2a

S2a (S2a-R1 compatibility) ${ }^{1}$ over each SOA, and submitted the mean Task 1 RTs to a 2 (R1-R2 compatibility) $\times 2$ (S2a$\mathrm{R} 1$ compatibility) $\times 4(\mathrm{SOA})$ repeated measures ANOVA with all factors considered within subjects. For Task 2, we again evaluated R1-R2 compatibility between tasks and also assessed within-task response compatibility between the to-be-ignored S2a and R2 (S2a-R2 compatibility). Mean RTs were submitted to a 2 (R1-R2 compatibility) $\times 2$ (S2a$\mathrm{R} 2$ compatibility) $\times 4(\mathrm{SOA})$ repeated measures ANOVA, again treating all factors as within subjects.

Error data were evaluated separately for each task. Trials with an error committed on Task 1, regardless of Task 2 accuracy, were assessed with respect to Task $1 \mathrm{R} 1-\mathrm{R} 2$ and S2a-R1 compatibility conditions, akin to RT data. Trials with an error committed on Task 2 after accurate Task 1 performance were assessed according to Task $2 \mathrm{R} 1-\mathrm{R} 2$ and S2a-R2 compatibility conditions, again akin to RT data. Participants' error rate data for each task were submitted to the same separate three-way repeated measures ANOVAs as that used for the RT analyses.

Finally, to directly test whether S2a-R2 effects observed in Task 2 were the result of indirect locus of slack effects from Task 1 or, instead, suggestive of S2a response information bypassing the bottleneck and directly influencing overt Task 2 performance, we conducted an analysis with data from short SOAs, using the difference between RT1 and RT2 as the dependent measure. For every trial with correct Task 1 and Task 2 performance, the RT for Task 1 was subtracted from that for Task 2 , and mean adjusted RT2 data were submitted to a 2 (R1-R2 compatibility) $\times 2$ (S2a$\mathrm{R} 2$ compatibility) $\times 2(\mathrm{SOA})$ repeated measures ANOVA.

\footnotetext{
${ }^{1}$ We chose our compatibility labels to distinguish between the compatibility between two executed responses (R1-R2, responses here made to $\mathrm{S} 1$ and $\mathrm{S} 2 \mathrm{~b}$ ) and the compatibility between a stimulus that did not require a response and an executed response (i.e., S2a-R1 and $\mathrm{S} 2 \mathrm{a}-\mathrm{R} 2)$. S2a-R2 compatibility here is analogous to the "congruence" manipulation in Schubert et al. (2008).
} 


\section{Results}

One participant was excluded from the analysis for failure to meet the accuracy criterion of $70 \%$ correct trials. RT trimming removed an average of $2.4 \%$ of trials from each of the remaining 19 participants' data. Mean RTs for the remaining trials with correct Task 1 and Task 2 performance are displayed in Fig. 3.

\section{Task 1 reaction time}

The analysis revealed no main effect of S2a-R1: Mean RTs did not differ across S2a-R1 compatibility conditions, $F(1$, $18)<1$. Additionally, the main effect of R1-R2 compatibility did not reach significance, $F(1,18)=2.12, p=.16$. However, we found an interaction between these two factors, $F(1,18)=$ 28.40, $M S E=772.28, p<.001$, demonstrating that RT1 was indeed influenced by compatibility with both S2a and R2. Performance was fastest in Task 1 for trials where the theoretical response to $\mathrm{S} 2 \mathrm{a}$ and executed response to $\mathrm{S} 2 \mathrm{~b}$ (i.e., R2) were both compatible or both incompatible with R1 (black lines in the left panel of Fig. 3) and slower for trials with mixed Task 2-to-Task 1 compatibility (where the response to only one of the Task 2 stimuli was compatible with R1, the response associated with either $\mathrm{S} 2 \mathrm{a}$ or $\mathrm{R} 2$, but not both [gray lines in the left panel of Fig. 3]). These observations were confirmed by the simple main effects: When R1 and R2 were compatible (solid lines in the left panel of Fig. 3), S2a-R1 compatible trials were faster $(M=693.00, S D=146.60)$ than $\mathrm{S} 2 \mathrm{a}-\mathrm{R} 1$ incompatible trials $(M=710.70, S D=152.52)$, $t(18)=-4.35, p<.001$; however, when R1 and R2 were incompatible (dashed lines), the reverse was true (S2a-R1 compatible, $M=716.04, S D=156.23$; $\mathrm{S} 2 \mathrm{a}-\mathrm{R} 1$ incompatible, $M=699.76, S D=150.51), t(18)=3.13, p=.006$. Thus, despite the fact that participants did not execute a response to the distractor $\mathrm{S} 2 \mathrm{a}$, it still had an influence on Task 1 processing through its interaction with R1-R2 compatibility. Additionally, there was some speeding of RT with increasing SOA, $F(3,54)=19.76, M S E=14,378.10, p<.001$.

\section{Task 2 reaction time}

Response compatibility effects were also observed in Task 2. Trials in which both the distractor (S2a) and target (S2b) colored squares signaled the same response for Task 2 (i.e., S2a-R2 compatible trials; black lines in the right panel of Fig. 3) were performed faster than incompatible trials (gray lines), $F(1,18)=75.02, M S E=2,619.66, p<.001$. This strong effect of S2a-R2 compatibility was underadditive with SOA, $F(3,54)=6.63, M S E=1,447.95, p=.001$. Paired $t$-tests indicated that S2a-R2 compatible trials were significantly faster than incompatible trials at each SOA, all ts $>3.15$. S2a-R2 compatibility also interacted with R1-R2 compatibility, $F(1,18)=6.31, M S E=2,259.96, p=.022$, such that the S2a-R2 compatibility effect was larger when $\mathrm{R} 1$ and R2 were compatible (solid lines in the right panel of Fig. 3) [65 ms, $t(18)=8.49, p<.001]$, relative to when $\mathrm{R} 1$ and R2 were incompatible (dashed lines in the right panel of Fig. 3) [37 ms, $t(18)=4.42, p<.001]$. Finally, consistent with typical PRP results, Task 2 RT decreased significantly
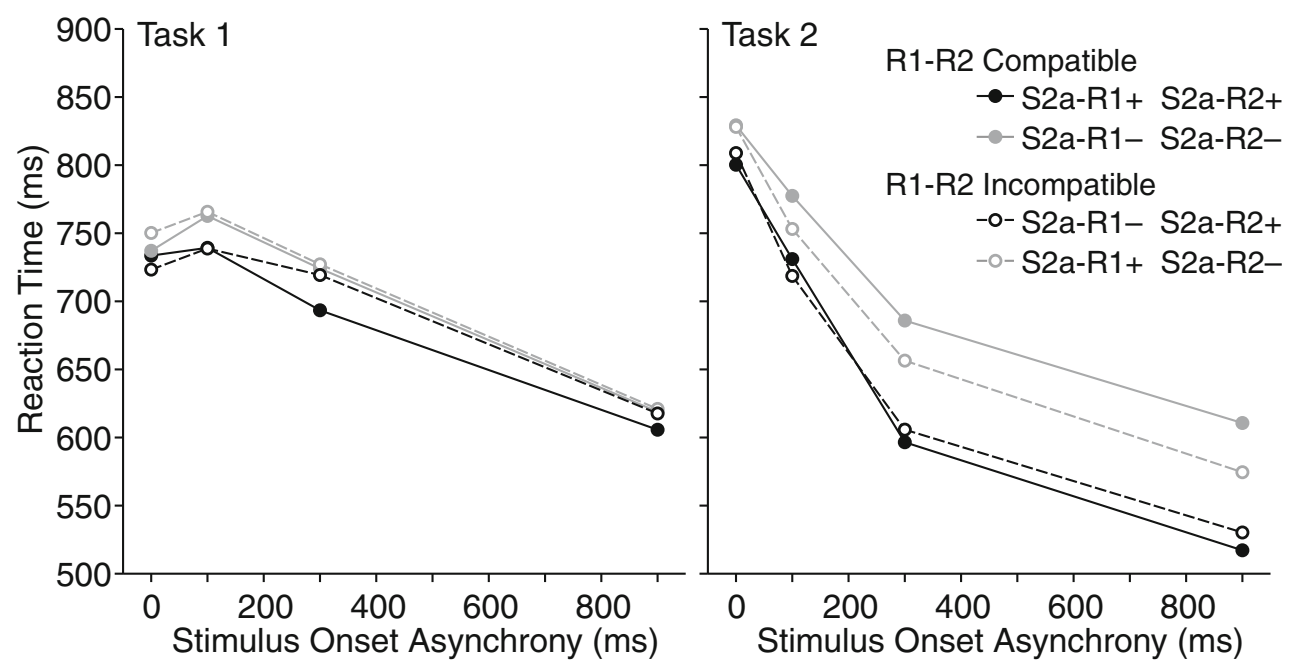

Fig. 3 Mean reaction time data for correct trials in Experiment 1. Data are divided by response compatibility relationships between executed responses in both tasks (R1-R2) and the S2a-related response compatibility relations (compatible, "“+”; incompatible, "“-") with the responses to Task 1 (S2a-R1) and Task 2 (S2a-R2). Note that for R1-R2 compatible trials, the $\mathrm{S} 2 \mathrm{a}$ compatibility relations must be the same for both tasks (e.g., S2a must be either compatible with both R1 and R2 or incompatible with

both). For R1-R2 incompatible trials, the S2a relations must differ across tasks (e.g., if S2a is compatible with R2, it must be incompatible with RT1, and vice versa). Task 1 data are discussed predominantly in terms of R1-R2 and S2a-R1 compatibility, while analysis of Task 2 data focuses on R1-R2 and S2a-R2 compatibility. Critical S2a-R2 compatibility effects are represented by black (compatible) versus gray (incompatible) lines for both Task 1 and Task 2 
with increasing SOA, $F(3,54)=173.75, M S E=5,736.35$, $p<.001$, with a slope approaching -1 across conditions at short SOAs.

\section{Errors}

Task 1 and Task 2 error rate data are presented in Table 1. In general, there were very few errors committed on Task 1 , or on both tasks in the same trial, whereas over $80 \%$ of all errors were committed on Task 2 following correct Task 1 performance. There were no significant effects involving Task 1 errors. Task 2 error data were largely consistent with the RT data. Performance was more accurate on compatible, relative to incompatible, S2a-R2 trials, $F(1,18)=21.01, M S E=.005$, $p<.001$. A main effect of R1-R2 compatibility demonstrated less accurate performance for compatible than for incompatible trials, $F(1,18)=5.27, M S E=.006, p=.034$. These two main effects were modified by an interaction between these factors, $F(1,18)=7.87, M S E=.002, p=.012$. Paired $t$-tests of simple main effects demonstrated that the effect of S2a-R2 compatibility was significant when R1 and R2 were compatible, $t(18)=6.34, p<.001$, and marginally significant when $\mathrm{R} 1$ and R2 were incompatible, $t(18)=2.05, p=.056$.

\section{Additional analysis of $S 2 a-R 2$ effects at short SOAs}

Adjusted RT2 measures for each condition in Experiment 1 are displayed in the top panel of Fig. 4. The analysis of RT2 minus RT1 data revealed a pattern of data quite similar to that observed in the original RT2 scores. This included a main effect of SOA, $F(1,18)=179.34, M S E=1,608.91, p<.001$, demonstrating that there was a larger difference between RT2 and RT1 at the 0 -ms SOA, as compared with the 100-ms SOA, indicative of a typical PRP effect. Critically, we still observed a main effect of S2a-R2 compatibility, $F(1,18)=11.13, M S E=$ $496.59, p=.004$, where $\mathrm{S} 2 \mathrm{a}-\mathrm{R} 2$ compatible trials (black lines in the top panel of Fig. 4) were faster than incompatible trials (gray lines) across both SOAs, with no interaction of S2a-R2 and SOA, $F(1,18)=0.90$. While there was no main effect of R1-R2 compatibility, $F(1,18)=1.40, p=.252$, R1-R2 compatibility was observed to interact with S2a-R2 compatibility, $F(1,18)=6.64, M S E=845.83, p=.019$. Analysis of simple effects showed that the effect of S2a-R2 compatibility was significant when R1 and R2 were compatible (solid lines) [24 ms, $t(18)=3.92, p=.001]$, but not when R1 and R2 were incompatible (dashed lines) [0 ms, $t(18)=0.02, p=.986]$. Additionally, observed differences in R1-R2 compatibility over SOA were also significant, $F(1,18)=5.05, M S E=$ 902.71, $p=.037$, because at the 100-ms SOA only, R1-R2 compatible trials were slower than incompatible trials, $t(18)=$ $2.93, p=.009$. The three-way interaction was not significant, $F(1,18)=1.24, p=.281$. This analysis suggests that when the responses made to each task were compatible, response information from S2a directly influenced RT2. However, when R1 and R2 were incompatible, the observed effect of S2a on RT2 was indirectly propagated from Task 1 via locus of slack mechanisms at short SOAs.

\section{Discussion}

The results of Experiment 1 demonstrate Task 2 to Task 1 response priming, replicating similar effects found by Hommel (1998), Watter and Logan (2006), Miller (2006), and others. This backward response priming effect on RT1 was

Table 1 Mean error rate (\% Error) and standard error of the mean (SEM) for each task in Experiment 1

\begin{tabular}{|c|c|c|c|c|c|c|c|c|c|c|}
\hline \multirow[t]{2}{*}{ SOA } & & & \multicolumn{2}{|l|}{0} & \multicolumn{2}{|l|}{100} & \multicolumn{2}{|l|}{300} & \multicolumn{2}{|l|}{900} \\
\hline & & & $\%$ Error & SEM & $\%$ Error & SEM & $\%$ Error & SEM & $\%$ Error & $S E M$ \\
\hline \multicolumn{11}{|c|}{ Task 1} \\
\hline & $\mathrm{R} 1-\mathrm{R} 2+$ & $\mathrm{S} 2 \mathrm{a}-\mathrm{R} 1+$ & 1.51 & 0.00 & 1.53 & 0.00 & 0.84 & 0.00 & 1.05 & 0.00 \\
\hline & & S2a-R1- & 0.99 & 0.01 & 1.06 & 0.00 & 0.84 & 0.00 & 0.17 & 0.00 \\
\hline & R1-R2- & $\mathrm{S} 2 \mathrm{a}-\mathrm{R} 1+$ & 1.55 & 0.01 & 1.01 & 0.01 & 1.59 & 0.01 & 0.58 & 0.00 \\
\hline & & S2a-R1- & 1.21 & 0.00 & 1.68 & 0.01 & 0.33 & 0.00 & 1.17 & 0.00 \\
\hline \multicolumn{11}{|c|}{ Task 2} \\
\hline & R1-R2+ & $\mathrm{S} 2 \mathrm{a}-\mathrm{R} 2+$ & 4.37 & 0.01 & 5.04 & 0.01 & 2.18 & 0.01 & 4.06 & 0.01 \\
\hline & & S2a-R2- & 8.42 & 0.01 & 9.67 & 0.01 & 10.59 & 0.02 & 7.50 & 0.02 \\
\hline & R1-R2- & $\mathrm{S} 2 \mathrm{a}-\mathrm{R} 2+$ & 4.88 & 0.01 & 3.23 & 0.01 & 2.83 & 0.01 & 2.62 & 0.01 \\
\hline & & $\mathrm{S} 2 \mathrm{a}-\mathrm{R} 2-$ & 5.44 & 0.02 & 5.73 & 0.02 & 5.62 & 0.02 & 5.61 & 0.02 \\
\hline
\end{tabular}

Note. Task 1 data are divided by response compatibility relationships (compatible, “+”; incompatible, “-“) on overt Task 1 performance from the S2b-related overt Task 2 response (R1-R2) and S2a-related response information (S2a-R1) and stimulus onset asynchrony (SOA). Task 2 data are divided by the response compatibility relationships on overt Task 2 performance from the S1-related overt Task 1 response (R1-R2) and S2a-related response information (S2a-R2) and SOA. The Task 1 error rates presented are independent of Task 2 performance, while Task 2 error rates are those observed after accurate Task 1 performance 

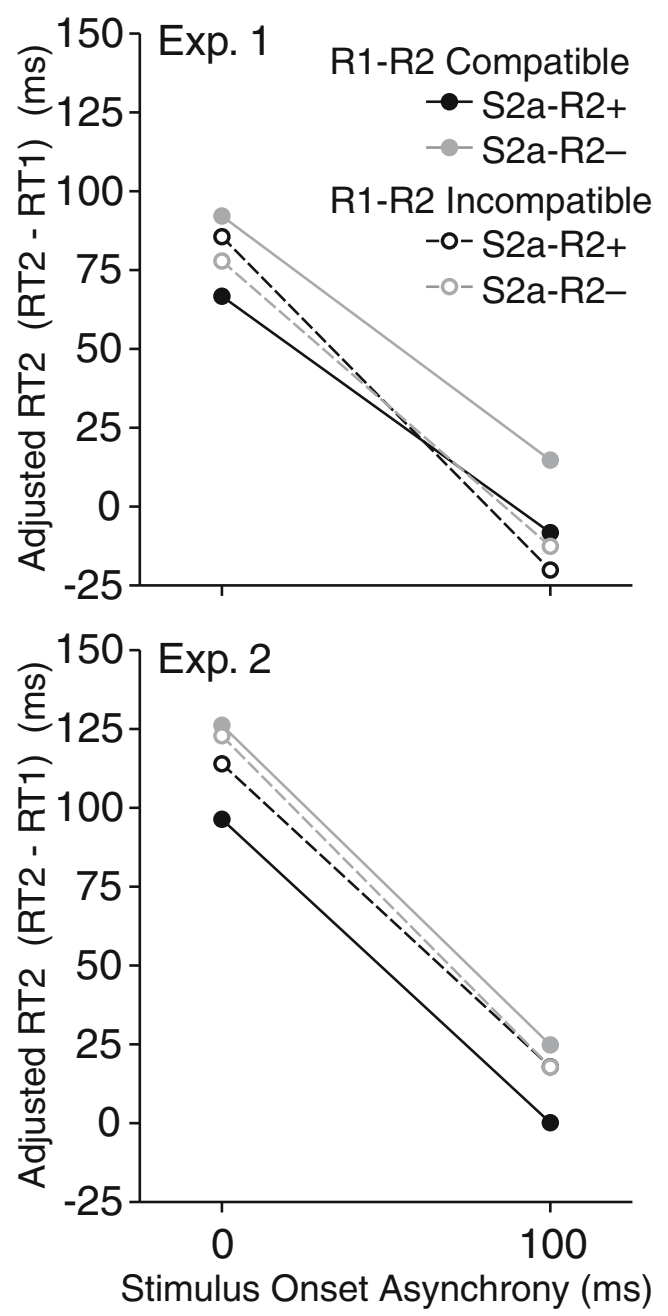

Fig. 4 Mean adjusted Task 2 reaction time (RT) data for correct trials at short SOAs for Experiments 1 and 2. Task 1 RT was subtracted from Task 2 RT for each trial, in order to control for any indirect effects from Task 1 prebottleneck or bottleneck stages propagating onto Task 2 RT. Both experiments demonstrate significant S2a-R2 compatibility effects (compatible, "+", black lines; incompatible, “-“, gray lines) persisting in these adjusted RT2 scores, for trials on which R1 and $\mathrm{R} 2$ are compatible. This suggests that $\mathrm{S} 2 \mathrm{a}$-related response information persists across the response selection bottleneck to directly influence overt Task 2 performance for response-compatible but not responseincompatible trials, in both bivalent (Experiment 1) and univalent (Experiment 2) response situations

demonstrated for response information arising from the target S2b and, importantly also for response information from the transient distractor $\mathrm{S} 2 \mathrm{a}$, as demonstrated by the interaction of R1-R2 compatibility effects and S2a-R1 compatibility effects. This suggests that response information was computed for S2a in parallel with Task 1 performance, even though this stimulus was not predictive of the eventual Task 2 response and did not require a response itself. We suggest that under these conditions, and with experimental instructions prioritizing Task 1 performance, participants were unlikely to try to make strategic use of the transient S2a stimulus.
Task 1 performance was observed to be slower for trials with short SOAs when S2a and S2b were visible during Task 1 processing. This slowing could be indicative of parallel response selection (Navon and Miller, 2002; Tombu \& Jolicoeur, 2003) or simply indicate that participants were distracted, either by the color change or by the mere presence of another stimulus on the screen while they performed Task 1. There is little evidence to suggest that participants were strategically grouping their responses at short SOAs, since interresponse intervals (IRIs) averaged $284 \mathrm{~ms}$ over these trials and only $13 \%$ of those trials had an IRI less than $150 \mathrm{~ms}$. Omitting these trials from the adjusted RT2 analysis did not change the pattern of results.

We note that the RT1 data also show an interesting and initially counterintuitive interaction with respect to mixtures of response compatibility. Combinations of RT compatibility effects show relatively fast responding not only for cases where both relationships are response compatible, but also for cases where both relationships are incompatible. Cases with a mixture of one compatible and one incompatible relationship typically show greater RT costs. These kinds of "mixed compatibility" costs have been previously observed in similar studies by the present authors using PRP tasks (e.g., Thomson et al., 2010; Watter \& Logan, 2006). These effects are explored more fully in other work (e.g., Hommel's [2004, 2007] work on event files and partial match effects, and we refer the reader there for further explanation. It is worth noting, however, that for our present analysis, trials that were assessed as fully compatible or fully incompatible and produced the fastest responses in Task 1 are also by definition S2a-R2 compatible trials. The slower mixed compatibility trials are, in fact, trials where $\mathrm{S} 2 \mathrm{a}$ and $\mathrm{S} 2 \mathrm{~b}$ are incompatible in Task 2 (for example, if the responses associated with $\mathrm{S} 1$ and $\mathrm{S} 2 \mathrm{a}$ are compatible but R1 and R2 are incompatible, then S2a and R2 must also be incompatible). Schubert et al. (2008) analyzed their Task 1 data according to these Task 2 compatibility relationships and observed an effect of S2a-R2 compatibility in Task 1. It is possible that $\mathrm{S} 2 \mathrm{a}-\mathrm{R} 2$ compatibility itself has an influence on the length of the response selection stage at short SOAs in Task 1, independently of the partial match effects described above (see Schubert et al.'s, 2008, discussion of Experiment 2 for a proposed mechanism for this manner of cross talk). The presence of this cross talk is not the focus of our analysis; however, it does further necessitate the subsequent analysis of adjusted RT2 scores, as is discussed below.

Central to our investigation is whether response information generated from S2a in parallel with attended Task 1 performance persisted across the bottleneck, surviving the attentional switch from Task 1 to Task 2 performance, to directly influence RT2. We observed a compatibility effect in our initial analysis of RT2 between the associated (but 
unmade) response to S2a and the attended, S2b-driven Task 2 response $\mathrm{R} 2$ ( $2 \mathrm{a}-\mathrm{R} 2$ compatibility). However, it is possible that this effect may have been due to the indirect influence of either $\mathrm{S} 2 \mathrm{a}-\mathrm{R} 1$ or $\mathrm{S} 2 \mathrm{a}-\mathrm{R} 2$ compatibility effects at prebottleneck or bottleneck stages in RT1, influencing RT2 at short SOAs via locus of slack effects. Schubert et al. (2008) argued that S2a influences on RT2 operated via this indirect influence and that S2a-related response information did not survive across the bottleneck to directly influence Task 2 performance.

To determine whether this was the case in our study, we conducted an additional analysis on RT2 minus RT1 difference scores, calculated for every trial at the shortest two SOAs, where we observed a strong PRP effect. Adjusting RT2 in this way should remove any effects influencing Task 1 prebottleneck or bottleneck stages, so that any cross talk or compatibility effects from Task 2 on Task 1 that propagate via locus of slack onto Task 2 RTs are removed from the resultant adjusted RT2 scores. If S2a exerted an influence on Task 2 only via this indirect mechanism, the S2a-R2 compatibility effect should disappear in this analysis. We still observe a strong influence of S2a-R2 compatibility on these adjusted Task 2 RT scores, but only when R1 and R2 were also compatible. When the responses across tasks were incompatible, the adjusted RT2 scores do not demonstrate the S2a-R2 compatibility effect.

One possible explanation is that the bypass of S2a information across the bottleneck is robust only within a single response effector (in this case, the same finger). When participants use one finger to respond to Task 1 and a different finger to respond to Task 2, perhaps any currently activated response information is reset when it becomes apparent that the second task requires a response that is different from the one executed previously-including accumulated S2a response information, regardless of its compatibility with R2. This is consistent with the fact that Schubert et al. (2008) did not find evidence for the bypass model when participants responded to each task with a different hand, precluding response repetition. We address this issue in Experiment 2.

The results of Experiment 1 still have important implications for information continuity across the RSB, suggesting that $\mathrm{S} 2 \mathrm{a}-\mathrm{R} 2$ compatibility effects are not exclusively due to indirect effects via Task 1 . Taken together with our previous analysis that demonstrated an effect of S2a response information on Task 1, it appears that response information for $\mathrm{S} 2 \mathrm{a}$ is generated in parallel with attended Task 1 performance and, in certain situations, persists to at least some degree across the RSB to prime the overt response to the target stimulus in Task 2.

The S2a-R2 response compatibility effect in Task 2 from our main RT analysis was observed to be underadditive with SOA. Interpreted from a strict locus of slack perspective (e.g., Pashler, 1994), our data would suggest that the computation of response information can occur in parallel for both tasks in a PRP paradigm, prior to the observed central bottleneck on explicit Task 2 performance. We consider the implications of these data more fully under locus of slack and alternative models below, in the General Discussion section.

\section{Experiment 2}

In Experiment 1, we demonstrated that early response information for Task 2 can indeed bypass the bottleneck to influence later Task 2 response selection processing. These findings are in contrast to those of Schubert et al. (2008), who found only indirect propagation across the RSB. One important difference between these studies is that in our present Experiment 1, responses were performed with the same hand, using the same response keys across tasks. Using this bivalent design, we observed a bypass effect only when the responses were compatible across tasks, resulting in response repetition. It is possible that on response alternation trials, any prior response activation is ignored to prevent response perseveration. This would result in any accumulated response information for S2a being discarded between tasks. Since a two-handed, univalent design always results in response alternations between tasks, it seems plausible that the bivalent nature of our Experiment 1 design was the reason we found evidence in support of the bypass model when Schubert et al., with their univalent design, did not.

In order to test this possibility, we conducted a second experiment that replicated the univalent, two-handed response mapping used by Schubert et al. (2008). Participants performed the same tasks as in Experiment 1, but this time they responded to $\mathrm{S} 1$ with the index or middle finger of their right or left hand and responded to $\mathrm{S} 2 \mathrm{~b}$ with the index or middle finger of the opposite hand. If the bypass effect depends on response repetition and this is the reason that our results differ from those of Schubert et al., we should not observe any evidence of S2a information continuity across the RSB in Experiment 2. Instead, we should replicate the findings of Schubert et al. and observe that the only influence of early Task 2 response information on Task 2 performance is mediated indirectly by cross talk via Task 1 . Specifically, the S2a-R2 compatibility effect should have the same magnitude in Task 1 and Task 2, regardless of R1R2 compatibility, and therefore there should be no effect of S2a-R2 compatibility in the adjusted RT2 scores. Alternatively, observing evidence of direct S2a-R2 influences on RT2 as in Experiment 1 would indicate that response information for Task 2 can bypass the bottleneck even when the responses are not identical across tasks. 


\section{Method}

\section{Participants}

Twenty-eight undergraduate students (13 females) enrolled in psychology courses at McMaster University participated in the experiment in exchange for partial course credit. All had normal or corrected-to-normal vision, and all but 2 were right-handed.

\section{Apparatus, stimuli, and procedure}

The apparatus, stimuli, and procedure were identical to those in the previous experiment, except for the following changes. (1) A subset of participants completed the experiment on an HP Pro 3130 computer with a Samsung SyncMaster B2240 monitor. The same experimental software was used on both computers, and the stimuli were the same physical size on both monitors. (2) Participants made separate responses to each task using a different hand. Half of the participants responded to the letter task using the middle and index finger of their left hand on two response buttons located on the left side of the keyboard, while the other half responded to the letter task using the index and middle finger of their right hand on response buttons on the right side of the keyboard. Participants used the index and middle finger of the opposite hand to respond to the color task. Stimulus-response mapping was counterbalanced within each task. Since participants made responses to each task with a separate hand, we defined R1-R2 compatible trials as those that required the same finger across tasks (i.e., both index fingers or both middle fingers) and R1-R2 incompatible trials as those that required a different finger for each task (e.g., left middle finger and right index finger). ${ }^{2}$

\section{Results}

Two participants were excluded from the analysis because they did not meet the accuracy criterion of $70 \%$ correct trials. A third participant was excluded because he was unable to follow instructions to prioritize Task 1: Consistent response grouping resulted in very long RT1s in the 900-ms SOA condition, leaving no more than six trials per condition

\footnotetext{
${ }^{2}$ Note that in Schubert et al. (2008), R1-R2 compatibility was defined using spatially compatible fingers across tasks (left-middle/right-index fingers and left-index/right-middle fingers were compatible). This was important for them because they used a spatial Task 2 (directional arrows). The stimuli in the present experiment do not have such strong spatial associations, and we predicted finger identity would be a stronger source of compatibility than spatial compatibility in our tasks (see Watter \& Logan, 2006, for another demonstration of R1-R2 compatibility based on compatible fingers rather than spatial compatibility across hands).
}

under our RT trimming criteria. RT trimming for the remaining 25 participants removed an average of $3.6 \%$ of trials from each participant's data set. Mean RTs for correct responses are displayed in Fig. 5.

\section{Task 1 reaction time}

Observed variability in Task 1 RTs across SOAs produced a main effect of this variable, $F(3,72)=7.63, M S E=12,263.07$, $p<.001$. More important, the interaction of $\mathrm{S} 2 \mathrm{a}-\mathrm{R} 1$ and $\mathrm{R} 1-$ R2 compatibility approached significance, $F(1,24)=3.03$, $M S E=2,660.78, p=.094$, and there was a significant threeway interaction involving SOA, S2a-R1 compatibility, and R1-R2 compatibility, $F(3,72)=3.24, M S E=1,900.73, p=$ .027 . We therefore analyzed the two compatibility manipulations at each level of SOA and found only a significant interaction of S2a-R1 and R1-R2 compatibility at the 100ms SOA, $F(1,24)=21.67, M S E=851.24, p<.001$. Decomposing this interaction further revealed a pattern similar to that found in Experiment 1: Performance at this SOA was faster for trials in which the responses associated with both S2a and R2 were either compatible or incompatible with $\mathrm{R} 1$ than for trials with mixed compatibility. These observations are supported by the simple main effects. When R1 and R2 were compatible (solid lines in left panel of Fig. 5), S2a$\mathrm{R} 1$ compatible trials were marginally faster $(M=746.57, S D=$ 137.37) than S2a-R1 incompatible trials $(M=762.89, S D=$ $148.04), t(24)=1.99, p=.058$. However, when R1 and R2 were incompatible (dashed lines), S2a-R1 incompatible trials were faster $(M=736.89, S D=138.43)$ than $\mathrm{S} 2 \mathrm{a}-\mathrm{R} 1$ compatible trials $(M=774.89, S D=137.03), t(24)=-3.43, p=.002$. Again, this is analogous to the interpretation of a main effect of S2a-R2 compatibility (black vs. gray lines) producing faster RTs in Task 1, at least at the 100-ms SOA.

\section{Task 2 reaction time}

In Task 2, we again observed a main effect of S2a-R2 compatibility, where compatible trials (black lines in the right panel of Fig. 5) were performed significantly faster than incompatible trials (gray lines), $F(1,24)=103.66, M S E=$ $2,953.69, p<.001$. This effect of compatibility of distractor and target Task 2 stimuli was again underadditive with SOA, $F(3,72)=13.26, M S E=1,463.39, p<.001$. Paired samples $t$-tests indicated that the effect of S2a-R2 compatibility was significant at each SOA (all $t \mathrm{~s}>4.61$ ). In addition, the effect of $\mathrm{R} 1-\mathrm{R} 2$ response compatibility across tasks was marginally significant, $F(1,24)=3.60, M S E=1,533.81, p=.070$, with response compatible trials (solid lines in the right panel of Fig. 5) faster than response incompatible trials (dashed lines). Consistent with typical PRP data, Task 2 RTs decreased significantly with SOA, $F(3,72)=189.07$, $M S E=7,376.63, p<.001$. 

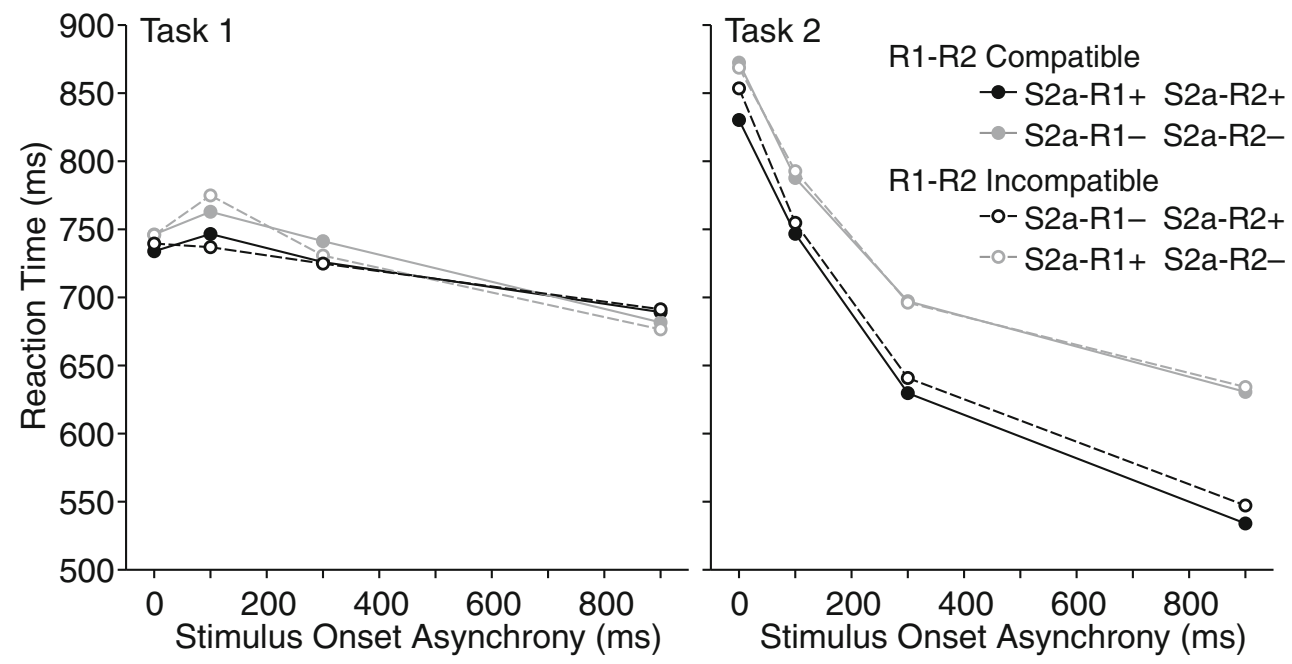

Fig. 5 Mean reaction time (RT) data for correct trials in Experiment 2. As in Fig. 3 (Experiment 1), data are divided by response compatibility relationships between executed responses in both tasks (R1-R2) and the S2a-related response compatibility relations (compatible, "+"; incompatible, "-") with the responses to Task 1 (S2a-R1) and Task 2 (S2a-R2). Note that for R1-R2 compatible trials, the S2a compatibility relations must be the same for both tasks (e.g., S2a must be either compatible with both R1 and R2 or incompatible with both). For R1-

\section{Errors}

Task 1 and Task 2 error data are presented in Table 2. Once again, there were no significant effects involving Task 1 errors. Task 2 accuracy data demonstrated that participants made fewer errors when S2a and R2 were compatible, $F(1$, 24) $=29.90, M S E=0.009, p<.001$, with this $\mathrm{S} 2 \mathrm{a}-\mathrm{R} 2$ effect more prominent at longer SOAs, $F(3,72)=14.10, M S E=$ $0.003, p<.001$. Finally, the interaction between R1-R2 compatibility and SOA approached significance, $F(3,72)=$ 2.47, MSE $=.002, p=.069$. This describes the pattern that $\mathrm{R} 1-\mathrm{R} 2$ compatible trials were numerically more accurate than R1-R2 incompatible trials at all but the 900-ms SOA.

\section{Additional analysis of $S 2 a-R 2$ effects at short SOAs}

We again calculated the difference between RT1 and RT2 for correct trials at the shortest two SOAs and conducted an analysis on these adjusted RT2 scores to investigate whether there was any influence of S2a on R2 independently of propagation of prebottleneck or bottleneck RT1 compatibility effects via locus of slack onto RT2. These adjusted RT2 data for Experiment 2 are shown in the bottom panel of Fig. 4. Once again, we observed a main effect of SOA, $F(1,24)=552.20$, $M S E=899.49, p<.001$, indicating that the difference between RT1 and RT2 was greater at the 0-ms SOA than at the 100-ms SOA. Critically, we again observed a main effect of S2a-R2 compatibility in the adjusted RT2 data, $F(1,24)=$ $8.55, M S E=1,465.79, p=.007$, and this effect did not interact
R2 Incompatible trials, the S2a relations must differ across tasks (e.g., if S2a is compatible with R2, it must be incompatible with RT1, and vice versa). Task 1 data are discussed predominantly in terms of R1$\mathrm{R} 2$ and $\mathrm{S} 2 \mathrm{a}-\mathrm{R} 1$ compatibility, while analysis of Task 2 data focuses on R1-R2 and S2a-R2 compatibility. Critical S2a-R2 compatibility effects are represented by black (compatible) versus gray (incompatible) lines for both Task 1 and Task 2

with SOA $(F<1)$. The S2a-R2 compatibility effect did interact with R1-R2 compatibility, $F(1,24)=6.72, M S E=$ $970.48, p=.016$. Analysis of the simple main effects demonstrated that the S2a-R2 compatibility effect (black vs. gray lines in the right panel of Fig. 4) was significant when R1 and $\mathrm{R} 2$ were compatible (solid lines) $[18 \mathrm{~ms}, t(24)=4.58$, $p<.001]$, but not when R1 and R2 were incompatible (dashed lines) $[-5 \mathrm{~ms}, t(24)=0.56, p=.58]$.

\section{Discussion}

The results of Experiment 2 largely replicated those of Experiment 1 . Task 1 responses were influenced by both R2- and S2a-related response information (although this effect reached significance only at the 100-ms SOA), and for RT2, we observed a strong influence of S2a-R2 compatibility that was underadditive with SOA. When we examined RT2 minus RT1 difference scores to assess whether S2a-R2 effects on RT2 were more than an indirect influence of effects propagating from RT1, we again found a significant effect of S2a-R2 compatibility on adjusted RT2 scores. As in Experiment 1, this effect was present only when R1 and R2 were compatible. This suggests that when responses were compatible across tasks, S2a-related response information was available to directly influence RT2 following the attentional shift from Task 1 to Task 2 performance.

These results suggest that early $\mathrm{S} 2 \mathrm{a}$ response information can bypass the bottleneck even when there is no physical response repetition, because in this experiment, the effect 
Table 2 Mean error rate (\% Error) and standard error of the mean (SEM) for each task in Experiment 2

\begin{tabular}{|c|c|c|c|c|c|c|c|c|c|c|}
\hline \multirow[t]{2}{*}{ SOA } & & & \multicolumn{2}{|l|}{0} & \multicolumn{2}{|l|}{100} & \multicolumn{2}{|l|}{300} & \multicolumn{2}{|l|}{900} \\
\hline & & & $\%$ Error & SEM & $\%$ Error & SEM & $\%$ Error & SEM & $\%$ Error & SEM \\
\hline \multicolumn{11}{|c|}{ Task 1} \\
\hline & $\mathrm{R} 1-\mathrm{R} 2+$ & $\mathrm{S} 2 \mathrm{a}-\mathrm{R} 1+$ & 3.31 & 0.01 & 3.54 & 0.01 & 2.24 & 0.01 & 2.32 & 0.01 \\
\hline & & S2a-R1- & 3.11 & 0.01 & 2.33 & 0.01 & 1.83 & 0.01 & 2.54 & 0.01 \\
\hline & R1-R2- & $\mathrm{S} 2 \mathrm{a}-\mathrm{R} 1+$ & 2.18 & 0.01 & 3.13 & 0.01 & 3.23 & 0.01 & 3.03 & 0.01 \\
\hline & & S2a-R1- & 3.86 & 0.01 & 3.32 & 0.01 & 2.54 & 0.01 & 2.61 & 0.01 \\
\hline \multicolumn{11}{|c|}{ Task 2} \\
\hline & $\mathrm{R} 1-\mathrm{R} 2+$ & $\mathrm{S} 2 \mathrm{a}-\mathrm{R} 2+$ & 8.58 & 0.02 & 6.90 & 0.02 & 4.56 & 0.01 & 4.81 & 0.01 \\
\hline & & S2a-R2- & 9.26 & 0.02 & 9.10 & 0.02 & 14.35 & 0.02 & 12.67 & 0.02 \\
\hline & R1-R2- & $\mathrm{S} 2 \mathrm{a}-\mathrm{R} 2+$ & 8.28 & 0.02 & 11.07 & 0.02 & 8.17 & 0.02 & 4.51 & 0.01 \\
\hline & & S2a-R2- & 11.24 & 0.02 & 10.40 & 0.02 & 14.99 & 0.03 & 11.94 & 0.02 \\
\hline
\end{tabular}

Note. Task 1 data are divided by response compatibility relationships (compatible, “+”; incompatible, '-') on overt Task 1 performance from the S2b-related overt Task 2 response (R1-R2) and S2a-related response information (S2a-R1) and stimulus onset asynchrony (SOA). Task 2 data are divided by the response compatibility relationships on overt Task 2 performance from the S1-related overt Task 1 response (R1-R2) and S2a-related response information (S2a-R2) and SOA. The Task 1 error rates presented are independent of Task 2 performance, while Task 2 error rates are those observed after accurate Task 1 performance.

was observed across different response hands. Our findings here are extremely similar to those in Experiment 1 and differ from those of Schubert et al. (2008), who did not observe information continuity across the bottleneck with a univalent design. It is worth noting that in this experiment, we did not observe an interaction of $\mathrm{R} 1-\mathrm{R} 2$ and $\mathrm{S} 2 \mathrm{a}-\mathrm{R} 2$ compatibility in our initial Task 2 analysis (Fig. 5). This replicates the results of Schubert et al., who found that these effects were independent and additive in Task 2. Despite this, we still observe an S2aR2 compatibility effect in our adjusted RT2 data. Notably, this bypass effect observed with our univalent design is again present only for R1-R2 compatible trials - in the present experiment, when responses are made with different effectors that are conceptually response compatible between tasks (based on finger identity, right-index-left-index or rightmiddle-left-middle). Taken together, these results might suggest a variable response resetting mechanism that suppresses Task 2 response information computed in parallel with Task 1 (and potentially Task 1 response information also) when responses are not compatible across tasks but does not completely suppress early response activation when responses are conceptually compatible. This possibility is explored further in the General Discussion section.

\section{General discussion}

Early Task 2 response activation can persist across the bottleneck

Despite several accounts of parallel response selection processes in dual-task paradigms, such studies have still exhibited substantial RT costs for Task 2 performance at short SOAs (Hommel, 1998; Miller, 2006; Miller \& Alderton, 2006; Thomson et al., 2010; Watter \& Logan, 2006). A number of authors have proposed models of dual-task performance that involve varying degrees of parallel computation of response information for both tasks at once, yet also involve serial final response selection prior to overt responding to each task (e.g., Hommel, 1998; Logan \& Gordon, 2001). An alternative possibility, discussed by Logan and Gordon and by Watter and Logan, is that any residual response information present following the eventual selection of a response for Task 1 might be discarded or suppressed in order to reduce interference on Task 2 performance. In this case, with sufficiently great suppression of any response information present at the end of Task 1 response selection, the overt engagement of response selection for Task 2 may essentially proceed with no persistent or residual activation from earlier in a trial, including an absence of any Task 2 response information that was generated in parallel with overt Task 1 response selection. In this case, the observation of Task 2 response compatibility effects on Task 1 performance would not necessarily violate the spirit of the RSB theory (Pashler, 1994; Pashler \& Johnston, 1998); in an informational sense, a strict bottleneck could be considered to be intact, in that Task 2 response selection would have to begin from scratch following the completion of Task 1 response selection.

In the present study, we set out to test the extent to which early Task 2 response information generated in parallel with attended Task 1 response selection could persist over the duration of a dual-task trial, to influence the eventual overt performance of Task 2. Two experiments employed a 
stimulus substitution technique in Task 2 to assess response priming from early unattended Task 2 response information on participants' eventual Task 2 responses. In both experiments, we found an influence of response information generated from both our early transient S2a stimulus and the task-relevant S2b stimulus on Task 1 performance that replicated similar findings of backward compatibility observed by Hommel (1998), Miller (2006), Miller and Alderton (2006), Thomson et al. (2010), and Watter and Logan (2006).

Critically, in both experiments, we demonstrated a clear influence of S2a-related response information on overt Task 2 performance at short SOAs, independent of any indirect influences from Task 1 prebottleneck or bottleneck processing stages propagating onto Task 2 . We suggest that this S2a-R2 effect represents direct evidence of early Task 2 response information generated in parallel with Task 1 response selection persisting across the attentional shift from Task 1 to Task 2 performance, bypassing the RSB and directly influencing overt response selection for Task 2. As an important boundary condition, we observed this effect only when Task 1 and Task 2 responses were compatible. In Experiment 1, this response compatibility existed as a repetition in Task 2 of the same physical response effector as that from Task 1 (bivalent; e.g., right-index-right-index); in Experiment 2, response compatibility was instantiated as responding with the same finger identity on the opposite hand for Task 2 as was used for Task 1 (univalent; e.g., right-index-left-index). When the responses were not compatible across tasks (e.g., right-index-right-middle in Experiment 1, right-index-left-middle in Experiment 2), we did not observe any influence of early Task 2 response information on overt Task 2 performance, beyond an effect that was indirectly propagated onto Task 2 via cross talk with Task 1 prebottleneck or bottleneck stages.

We contrast our findings with those of Schubert et al. (2008), who found only indirect effects of S2a-R2 compatibility on Task 2 performance (described there as congruency between the two Task 2 stimuli), mediated via compatibility effects on Task1 with propagation to RT2. Our data provide the first evidence supporting the bypass model, where early Task 2 response activation contributes directly to later, attended Task 2 response selection.

Our demonstration of equivalent S2a-related response compatibility effects across bivalent (Experiment 1) and univalent (Experiment 2) response mapping situations suggests that the locus of these effects (and backward response compatibility effects in general) is likely at the level of central and abstract response code representation, versus later response execution. The observation of response compatibility effects under differing R1-R2 compatibility definitions for the present study versus Schubert et al. (2008) is also consistent with this idea. Schubert et al. defined response compatibility by spatial correspondence, where we defined it as finger identity. Our tasks were explicitly described to participants in this way (describing response mappings as "index" or "middle" for particular stimuli); in contrast, many of Schubert et al.'s tasks used choice responding to spatial cues, and we speculate that there participants may have been instructed relative to "left versus right" responses within a hand, as opposed to finger identity. We note above (see footnote 2) that a number of prior studies have used both of these opposing versions of response compatibility (identity vs. spatial correspondence) and have generally found their own assumptions of compatibility confirmed. We suggest that our data combined with those in Schubert et al. demonstrate a somewhat flexible alignment of response compatibility relationships, based on task rules and the conceptualization of response features. We suggest that these observations provide further converging support for the notion of response compatibility effects influencing centrally represented response codes, as opposed to later response execution processes.

Variable resetting of response information between tasks

We are particularly interested in the observation that Task 1 to Task 2 (R1-R2) response compatibility appears to be a prerequisite for information continuity across the bottleneck for Task 2. We suggest that these data may represent the action of a response information resetting mechanism somewhat akin to the theoretical proposal of Logan and Gordon (2001), although here with a variable sensitivity depending on the degree of response compatibility between tasks.

Logan and Gordon (2001) and Watter and Logan (2006) suggested that while suppression of response information following Task 1 response selection would be important to prevent response perseveration and interference and allow for fast and accurate serial dual-task performance, the degree of suppression required to achieve this might be substantially less than $100 \%$. This would suggest any potential informational bottleneck instantiated via response suppression might be substantially leaky. Framed in this way, our current S2a-R2 compatibility data suggest an essentially complete informational bottleneck for response (R1-R2) incompatible trials but suggest that this response suppression is incomplete when Task 1 and Task 2 trials have compatible responses.

The question of how response compatibility might influence the degree of response information suppression on a trial-to-trial basis is a difficult one; in Logan and Gordon's (2001) treatment, response information suppression is treated as a global performance parameter. One possibility is that participants might become sensitive to the degree of response incompatibility experienced on each trial. At short SOAs, this interference would be substantially experienced 
prior to the completion of Task 1 response selection, from response information generated from Task 2 stimuli. The degree of conflict experienced in Task 1 response selection could directly drive the strength or extent of a subsequent response inhibition mechanism. In this situation, participants may learn (or put another way, this system may become tuned over performance) so that responsecompatible trials generating minimal response conflict on Task 1 response selection elicit relatively less response information suppression, in turn allowing some degree of response facilitation on response-compatible trials. This possibility may fit well with current literatures in response conflict and error monitoring (e.g., Yeung, Botvinick, \& Cohen, 2004) and may predict other correlates (such as anterior cingulate involvement), if true.

An alternative mechanism for this same effect may not require that response compatibility information directly drive response information suppression on a given trial. Instead, in an episodic account, particular stimulus pairs may become associated with the experience of relatively high or low degrees of response interference. Changes in the degree of response suppression would initially occur only after these experiences, but over time, these differing suppression states may be elicited directly from particular stimulus pairs prior to (or at least in parallel with) the activation and potential interference of response information for both tasks. This account is conceptually very similar to work by Bugg, Crump, and colleagues (Bugg, Jacoby \& Chanani, 2011; for a review, see Bugg \& Crump, 2012) showing rapid automatic contextual control of single-task performance with item-specific proportion congruency manipulations. A related possibility is that response information suppression occurs following a failure of event file integration (Hommel, 2004) on response-incompatible trials. These episodic accounts would predict the development of this variable response information suppression over experience, perhaps dependent on task rule complexity, while in the prior version, we might expect the degree of response information suppression to be immediate and more directly tied to transient activations within particular trials.

Considering all this, we suggest that the detection of direct S2a-R2 influences on RT2 should be considered as a marker for situations where we might expect the influence of other, stronger sources of response information to similarly persist across an informational bottleneck and influence RT2. We include here all response information generated prior to the beginning of Task 2 response selection - not just early Task 2 response activation, but response information from Task 1 also. There are substantial methodological difficulties in separating the direct influence of central Task 1 response information on RT2 from the motoric effects of an already-made R1 response on RT2; distinguishing the relative contributions of response information generated from a typical Task 2 stimulus prior to and during response selection is similarly tricky. Nevertheless, we suggest that these effects might be substantial and are predicted on the basis of the general assumption of response information suppression in serial dual-task performance and our demonstration of this variably permissive informational bottleneck.

Alternative models of response activation and response selection

In our demonstration of the direct influence of S2a-related response compatibility on overt Task 2 performance at short SOAs, S2a-R2 compatibility effects were observed to interact underadditively with SOA. Such underadditivity in RT in Task 2 of a PRP paradigm has traditionally been interpreted under the locus of slack logic (Pashler, 1994) as reflecting a task manipulation at a prebottleneck processing stage in Task 2. Framed in this way, our data suggest that the computation of response information may be embodied in a separate processing stage-here termed response activation, following Hommel (1998) - prior to the welldescribed central bottleneck involved with response selection. This interpretation is represented in the top half of Fig. 6 (panels a1 and a2). The duration of the response activation stage driven by the task-relevant $\mathrm{S} 2 \mathrm{~b}$ stimulus is relatively shorter when primed by compatible response information from the simultaneous response activation stage driven by the distractor $\mathrm{S} 2 \mathrm{a}$ stimulus ( $2 \mathrm{a}-\mathrm{R} 2+$ ), as compared with a longer duration of response activation for $\mathrm{S} 2 \mathrm{~b}$ when $\mathrm{S} 2 \mathrm{a}$ and $\mathrm{S} 2 \mathrm{~b}$ are associated with incompatible responses (S2a-R2-). At short SOAs (panel a1), these differences in response activation stage durations have little to no effect on overall S2b-driven Task 2 RT, since the later response selection stage for $\mathrm{S} 2 \mathrm{~b}$ is delayed until Task 1 response selection has finished. At longer SOAs (panel 2), the difference in $\mathrm{S} 2 \mathrm{~b}$ response activation stage duration due to compatibility effects from S2a response information have a greater influence on final Task $2 \mathrm{RT}$, since the final response selection stage is no longer delayed by Task 1 response selection.

While this interpretation may be initially appealing, the framing of response activation as a separate processing stage within the locus of slack framework would require a number of strict assumptions, including that response activation and selection processes were serially discrete and computationally independent. Many single-task models of central information processing do not make these assumptions and often make little distinction between the process of computing response information and the selection of a to-be-made response. For example, foundational work by Coles, Gratton, and colleagues (e.g., Coles, Gratton, Bashore, Eriksen, \& Donchin, 1985; Gratton, Coles, \& Donchin, 
A1
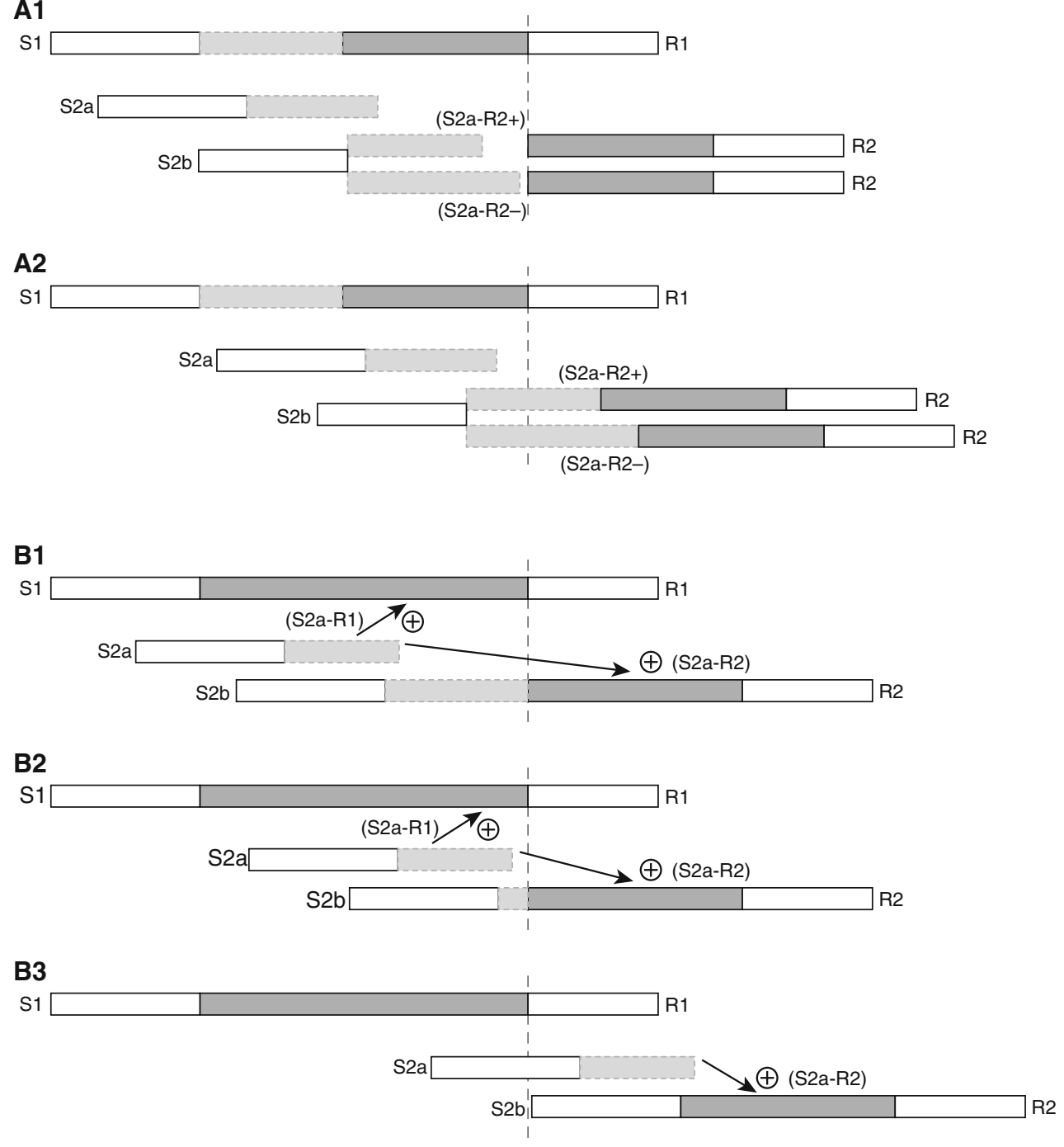

Fig. 6 Alternative models of underadditivity of S2a response compatibility effects on Task 2 performance (S2a-R2 compatibility) with SOA. Dark gray shading indicates the response selection stage; light gray shading indicates response activation. Panels a1 and a2 depict a strict locus of slack interpretation at shorter and longer SOAs, respectively. With a short SOA, the variable duration of the main S2b-related Task 2 response activation stage (due to compatibility effects from S2a response activation) is absorbed in prebottleneck slack time, with small or no differences in overall RT between S2a-R2+ (compatible) and S2a-R2(incompatible) conditions. At a longer SOA, overt response selection

1992; Gratton, Coles, Sirevaag, Eriksen, \& Donchin, 1988) suggests that while the computation and activation of a response may involve both parallel and focused processing stages, these stages are typically observed to be continuously cascaded, with contextual and strategic factors able to influence the degree to which parallel versus later focused processing stages contribute to determining the final response. Compatible with these ideas, work by Hommel and colleagues (e.g., Hommel, 2004; Hommel, Müsseler, Aschersleben, \& Prinz, 2001) and others suggests that with sufficient prior experience, associated response features are stages of Task 1 and Task 2 no longer overlap, and so differences in Task 2 response activation stage durations are fully reflected in overt Task 2 RTs. Panels b1, b2, and b3 represent response priming effects of early parallel response activation from $\mathrm{S} 2 \mathrm{a}$ in a non-serially dependent model of response activation, over progressively longer SOAs. Limited S2arelated response activation is increasingly separated in time from overt Task 2 response selection as SOA decreases. This separation could restrict the influence of early S2a response information at shorter, relative to longer, SOAs, leading to an underadditive pattern of S2a-R2 compatibility and SOA in the present study

automatically and directly activated from episodic memory by related perceptual cues, in the absence of deliberate focused response selection performance. We suggest that these kinds of effects, when embodied in a PRP-like dualtask situation, seem conceptually very similar to the effects of prebottleneck parallel response activation for Task 2 and related response compatibility effects suggested in the present article and elsewhere.

In a similar sense, one can suggest a number of situations where response activation and final response selection processes may have a reciprocal or interactive relationship 
within a single task. For example, in the present study as previously (e.g., Thomson et al., 2010; Watter \& Logan, 2006), early activation of Task 2 response information in parallel with overt Task 1 performance has been suggested to be unattended and automatic, while overt Task 2 performance (following Task 1 response selection) is assumed to be attended and controlled. If we consider unattended Task 2 response activation to operate along the lines of a general competitive information accrual model, (e.g., Nosofsky \& Palmeri's [1997] exemplar-based random walk model or a simpler race-style or diffusion-style model), then the time for which this automatic response activation process runs will influence the quality of response activation when overt Task 2 processes begin. Other things being equal, this might predict that when relatively little unattended response activation occurs prior to overt task performance, overt response selection may need to perform relatively more work than when unattended response activation has run for longer. While this issue is difficult to directly assess, the general point is that there is good reason to suspect that automatic response activation and overt response selection processes might be quite interdependent, with reciprocal relationships relative to computation of task responses.

The flexibility of overt response selection to perform more or less response computation, depending on the extent of unattended response activation or the degree of other potential influences (e.g., degrees of interference), is quite distinct from the situation discussed above where a separate response activation stage is incorporated into a locus of slack framework; in that case, response activation and response selection would need to assume serially distinct processing domains - for example, computation of response information in response activation and subsequent response gating in response selection.

Redundancy of processing in two sequential stages would also violate the seriality assumptions of the model. For example, having two sequential stages that both generate the same kind of information (e.g., both processes may increment the same response counters) is a very strange situation in terms of serial stage models commonly used in cognitive psychology. Similarly, response activation as a processing stage might be able to run concurrently with overt response selection, akin to various automaticity accounts (e.g., Logan, 1988), where algorithmic and instance-based processes race to produce a response. These characteristics make response activation a very difficult process to fit cleanly into a discrete serial stage model, when the process in question does not have to reach some asymptote of information processing before subsequent processes can begin. While processing stages are in reality likely cascaded and overlapping, most authors take the practical approach of assuming that a serial and discrete model is a sufficient approximation, due to computational and interpretational simplicity.
If dependencies between automatic response activation and attended response selection performance in Task 2 suggest that the serial stage locus of slack logic may be an inappropriate model for interpreting our findings, how should we alternatively interpret the underadditivity with SOA in our Task 2 response priming data? One suggestion is illustrated in the lower half of Fig. 6. The three lower panels illustrate a progression of the general time course of processing of our present design, over shorter through longer SOAs (panels b1-b3), with locus of slack assumptions only regarding the serial dependency of overt central response selection processing. The potential effects of response information generated from the distractor S2a on Task 1 (S2a-R1) and Task 2 (S2a-R2) responses are indicated. In the present set of experiments, S2a was presented for a short and constant period of time ( $200 \mathrm{~ms}$ ) prior to being replaced by the main task-relevant S2b stimulus, regardless of SOA. As such, at long SOAs, S2a was presented relatively close in time with overt Task 2 performance; as SOA decreased, overt Task 2 performance was delayed more and more, separating overt Task 2 performance from the initial transient S2a presentation. We suggest that any response activation arising from S2a would likely be time limited or would reach some maximal extent of activation relatively quickly in the course of the trial, especially given the short presentation and replacement with a task-relevant stimulus from the same set. As such, overt Task 2 performance at short SOAs may have been less influenced by S2a response information, due to increased separation in time from the activation of S2aderived response information, as compared with Task 2 performance at later SOAs. If the S2a distractor had remained onscreen for the duration of the trial, to continue to drive automatic response activation, such an underadditive result may not have been observed.

This argument so far assumes S2a-related response information to be generated in the same fashion at all SOAs, which is probably not the case. We suggest that S2a likely has additional pronounced effects on RT2 as SOA increases, when S2a is present onscreen during overt Task 2 performance following completion of Task 1 response selection, and complicates straightforward comparisons and predictions of S2a effects at early versus late SOAs. Bearing this caveat in mind, we note that the magnitude of our S2a-R2 compatibility effects at short SOAs is approximately one third the size of the S2a-R2 compatibility effect observed at the 900-ms SOA in both experiments. This suggests that while a detectable influence of S2a information might persist across the bottleneck, a majority of the S2a-related response information (or at least a majority of the measured compatibility effect) does not appear to survive to influence RT2. Early Task 2 response activation does appear to directly influence RT2, but the effect may be relatively small as compared with the contribution of overt Task 2 response selection, at least in these kinds of task 
situations. This said, we focus here on $\mathrm{S} 2 \mathrm{a}$ effects because we can distinguish $\mathrm{S} 2 \mathrm{a}-\mathrm{R} 2$ effects from the effect of producing $\mathrm{R} 2$ from the target stimulus S2b. It is possible that potentially much larger direct effects might arise from early Task 2 response activation from the target $\mathrm{S} 2 \mathrm{~b}$ stimulus, especially considering its continuous availability and task-relevancealthough quantifying the degree of response information generated from automatic $\mathrm{S} 2 \mathrm{~b}-\mathrm{R} 2$ activation versus overt $\mathrm{S} 2 \mathrm{~b}-$ $\mathrm{R} 2$ response selection is a more difficult problem.

\section{Alternative accounts of S2a-R2 compatibility effects}

Finally, we consider two potential alternative interpretations of our S2a to R2 compatibility effects. One alternative is that instead of representing the effects of response information generated from S2a on R2, these S2a-R2 compatibility effects may represent priming of prebottleneck semantic category representations. By this account, S2a would only facilitate the activation of the semantic category representation of S2b (when compatible), leading to faster Task 2 performance due to a presumably faster or stronger establishment of the S2b category representation, without any direct effect of S2a-related response information on RT2.

Compatibility between semantic category representations in dual-task situations akin to the present general PRP design have been shown to produce Task 2 to Task 1 priming effects (e.g., Logan \& Schulkind, 2000) that look extremely similar to the Task 2 to Task 1 response priming effects we claim to observe here. Watter and Logan (2006) and Thomson et al. (2010) deliberately employed methods to explicitly distinguish semantic category backward compatibility effects from response-related backward compatibility effects and demonstrated that both were present when using semantic categorization tasks where stimuli from both tasks were semantically meaningful and interpretable under both Task 1 and Task 2 categorization rules (e.g., magnitude and parity tasks on digits, or animacy and size judgments on words). As compared with these previous studies, we suggest that our present experiments employed tasks whose semantic categories were relatively weak and unpracticed, with respect to indicating alternative responses. Our tasks also had minimal semantic overlap (stimuli for one task were not semantically meaningful to the other task), suggesting that our observed Task 2 to Task 1 priming effects of S2a-R1 compatibility (and their interaction with R1-R2 compatibility) were likely due to response information arising from S2a. A purely semantic priming account of our observed S2a-R2 effects would have to assume that S2a-related response information was present during overt Task 1 performance (as measured by effects on Task 1) but was unable to directly influence later overt Task 2 response selection.

From a locus-of-slack perspective, a prebottleneck semantic S2a priming effect in Task 2 would be predicted to be underadditive with SOA. While we observe an obvious underadditive relationship across SOAs for S2a-R2 compatibility in our Task 2 data, we suggest that these conditions likely include additional priming effects of attended processing of S2a (with related single-task-like semantic and response process facilitation) at medium and long SOAs, where S2a is still onscreen when Task 1 response selection is complete (a nonnegligible proportion of trials at the 300-ms SOA and, most of the time, for the 900-ms SOA). At short SOAs, where we can more critically examine the unattended influence of S2a, we should predict either observing underadditivity if the prebottleneck Task 2 semantic categorization process still extends beyond the completion of Task 1 response selection to some degree (or still does so on some smaller proportion of trials) or no difference between S2a-R2 conditions if Task 2 semantic activation is maximal (in serial model terms, if the stage is complete) prior to the completion of Task1 response selection.

In contrast to these predictions of a prebottleneck locus for our S2a-R2 effect, our adjusted RT2 data (controlling for any indirect influence of early cross talk effects on RT1 propagated onto RT2 via locus of slack effects, as discussed above and by Schubert et al., 2008) show a substantial and consistent size of S2a-R2 compatibility effect at 0 - and 100ms SOAs for R1-R2 compatible trials and consistently no S2a-R2 compatibility effect on R1-R2 incompatible trials for these early SOAs. The presence and consistent size of our critical S2a-R2 compatibility effect on R1-R2 compatible trials is inconsistent with a prebottleneck Task 2 semantic locus of this effect. Further, the RSB model (Pashler, 1994; Pashler \& Johnston, 1998) predicts that a potential S2a prebottleneck semantic priming effect should not interact with response compatibility effects in a serially discrete response selection stage. Instead, our data show consistent and substantial interactions of S2a-R2 and R1-R2 compatibility relationships, again arguing against a prebottleneck semantic mediation account of our S2a-R2 effects.

A second possible alternative suggested to us is that $\mathrm{S} 2 \mathrm{a}$ response information itself may not bypass the bottleneck but, rather, that some representation of the S2a stimulus persists over time and its corresponding response information is later activated during attended Task 2 response selection. According to this alternative, the S2a-R2 compatibility effect observed in these experiments would not be in conflict with the traditional RSB theory because the generation of response information from a persisting S2a representation occurs after the bottleneck, rather than in parallel with Task 1 response selection. We suggest several arguments against this interpretation. If S2a produced only a perceptual or conceptual representation prior to the bottleneck, we might expect the strength of this representation to be relatively diminished when it is eventually used to derive response information during Task 2 response selection, in 
comparison with that of an S2a stimulus on the screen (which would occur well before overt Task 2 response selection at shorter SOAs). The benefit of computing response activation from S2a immediately is that that response activation can be generated and then persist as it is; in contrast, a persisting conceptual identity representation needs to subsequently drive the computation or selection of another processing stage, in the face of direct perceptual and attentional competition from another stimulus (S2b). Of course, such a possibility could be considered as a reason as to why S2a-R2 influences are relatively small.

Importantly, electrophysiological studies measuring lateralized readiness potentials have demonstrated that response activation for Task 2 does, in fact, begin before the completion of Task 1 response selection (e.g., Lien, Ruthruff, Hsieh, \& Yu, 2007; Logan, Miller, \& Strayer, 2011). While in our present case, these data most directly predict the early generation of $\mathrm{S} 2 \mathrm{~b}$ response information, we see no reason why these effects should not be generated by S2a in the exact same way. While we cannot conclusively discount the various alternative accounts described above, we suggest that a set of converging evidence from this and other studies is supportive of the notion of early response information generation in Task 2 and that our present data suggest that this information can directly influence overt Task 2 performance with appropriate response conditions.

\section{Conclusions}

In summary, while a number of recent studies have shown evidence for parallel activation of Task 2 response information while engaged with overt Task 1 response selection, these data may not violate the core principles of the RSB model (Pashler 1994; Pashler \& Johnston, 1998). If response information present following Task 1 response selection is suppressed, as some models suggest may be necessary to resolve interference and binding issues in dual-task performance (e.g., Logan \& Gordon, 2001), then attended Task 2 response selection would proceed without any influence of prior automatically activated Task 2 response information computed in parallel with Task 1 response selection. In this situation, Task 2 response selection could be said to be strictly informationally discrete from Task 1 response selection, preserving the essential nature of the RSB model. We present evidence that early Task 2 response information generated in parallel with overt Task 1 response selection does, in fact, persist throughout a trial and across the attentional switch from overt Task 1 to overt Task 2 performance and that this early parallelgenerated Task 2 response information influences overt Task 2 responding. In contrast to Schubert et al. (2008), who suggested that early Task 2 response activation influences RT2 only indirectly via propagation of RT1 prebottleneck and bottleneck cross talk effects onto RT2, our data suggest that early Task 2 response information can directly influence RT2, independently of these indirectly mediated effects, in both univalent and bivalent situations when Task 1 and Task 2 responses are compatible. This persistence of early parallel-generated Task 2 response information across the RSB suggests that traditional bottleneck models need modification and suggests some constraints on potential models of human central information processing.

Author Note We are grateful to members of McMaster University's Cognitive Science Laboratory for assistance with data collection and to Karin R. Humphreys for useful discussions about this work. This research was supported by a Natural Sciences and Engineering Research Council of Canada (NSERC) Grant \#327454 to S.W. and by an NSERC postgraduate scholarship (PGS-D) to S.J.T. Correspondence concerning this article should be sent to S. J. Thomson or S. Watter, Department of Psychology, Neuroscience \& Behaviour, McMaster University, 1280 Main Street West, Hamilton, L8S 4K1, Canada (email: thomsosj@mcmaster.ca or watter@mcmaster.ca).

\section{References}

Bugg, J. M., \& Crump, M. J. (2012). In support of a distinction between voluntary and stimulus-driven control: A review of the literature on proportion congruent effects. Frontiers in Psychology, 3, 367 .

Bugg, J. M., Jacoby, L. L., \& Chanani, S. (2011). Why it is too early to lose control in accounts of item-specific proportion congruency effects. Journal of Experimental Psychology. Human Perception and Performance, 37, 844-859.

Coles, M. G. H., Gratton, G., Bashore, T. R., Eriksen, C. W., \& Donchin, E. (1985). A psychophysical investigation of the continuous flow model of human information processing. Journal of Experimental Psychology. Human Perception and Performance, 11, 529-553.

Fischer, R., Kiesel, A., Kunde, W., \& Schubert, T. (2011). Selective impairment of masked priming in dual-task performance. The Quarterly Journal of Experimental Psychology, 64, 572595.

Gratton, G., Coles, M. G. H., \& Donchin, E. (1992). Optimizing the use of information: Strategic control of activation of responses. Journal of Experimental Psychology. General, 121, 480-506.

Gratton, G., Coles, M. G. H., Sirevaag, E. J., Eriksen, C. W., \& Donchin, E. (1988). Pre- and poststimulus activation of response channels: A psychophysiological analysis. Journal of Experimental Psychology. Human Perception and Performance, 14, 331344.

Hommel, B. (1998). Automatic stimulus-response translation in dualtask performance. Journal of Experimental Psychology. Human Perception and Performance, 24, 1368-1384.

Hommel, B. (2004). Event files: Feature binding in and across perception and action. Trends in Cognitive Sciences, 8, 494500.

Hommel, B. (2007). Feature integration across perception and action: Event files affect response choice. Psychological Research, 71, 42-63. 
Hommel, B., Müsseler, J., Aschersleben, G., \& Prinz, W. (2001). The theory of event coding (TEC): A framework for perception and action planning. Behavioural and Brain Sciences, 24, 849-937.

Lien, M.-C., Ruthruff, E., Hsieh, S., \& Yu, Y.-T. (2007). Parallel central processing between tasks: Evidence from lateralized readiness potentials. Psychonomic Bulletin \& Review, 14, 133-141.

Logan, G. D. (1988). Toward an instance theory of automatization. Psychological Review, 95, 492-527.

Logan, G. D., \& Gordon, R. D. (2001). Executive control of visual attention in dual-task situations. Psychological Review, 108, 393 434.

Logan, G. D., \& Schulkind, M. D. (2000). Parallel memory retrieval in dual-task situations: I. Semantic memory. Journal of Experimental Psychology. Human Perception and Performance, 26, 10721090.

Logan, G. D., Miller, A. E., \& Strayer, D. L. (2011). Electrophysiological evidence for parallel response selection in skilled typists. Psychological Science, 22, 54-56.

Meyer, D. E., \& Kieras, D. E. (1997a). A computational theory of executive cognitive processes and multiple-task performance: Part 1. Basic mechanisms. Psychological Review, 104, 3-65.

Meyer, D. E., \& Kieras, D. E. (1997b). A computational theory of executive cognitive processes and multiple-task performance: Part 2. Accounts of psychological refractory-period phenomena. Psychological Review, 104, 749-791.

Miller, J. (2006). Backward crosstalk effects in psychological refractory period paradigms: Effects of second-task response types on first-task response latencies. Psychological Research, 70, 484493.

Miller, J., \& Alderton, M. (2006). Backward response-level crosstalk in the psychological refractory period paradigm. Journal of
Experimental Psychology. Human Perception and Performance, 32, 149-165.

Navon, D., \& Miller, J. (2002). Queuing or sharing? A critical evaluation of the single-bottleneck notion. Cognitive Psychology, 44, 193-251.

Nosofsky, R. M., \& Palmeri, T. J. (1997). An exemplar-based random walk model of speeded classification. Psychological Review, 104, 266-300.

Pasher, H. (1984). Processing stages in overlapping tasks: Evidence for a central bottleneck. Journal of Experimental Psychology. Human Perception and Performance, 10, 358-377.

Pashler, H. (1994). Dual-task interference in simple tasks: Data and theory. Psychological Bulletin, 116, 220-244.

Pashler, H., \& Johnston, J. C. (1998). Attentional limitations in dualtask performance. In H. Pashler (Ed.), Attention (pp. 155-189). Hove: Psychology Press.

Schubert, T., Fischer, R., \& Stelzel, C. (2008). Response activation in overlapping tasks and the response-selection bottleneck. Journal of Experimental Psychology. Human Perception and Performance, 34, 376-397.

Thomson, S. J., Watter, S., \& Finkelshtein, A. (2010). Parallel response selection in dual-task situations via automatic category-to-response translation. Attention, Perception, \& Psychophysics, 72, 1791-1802.

Tombu, M., \& Jolicoeur, P. (2003). A central capacity sharing model of dual-task performance. Journal of Experimental Psychology. Human Perception and Performance, 29, 3-18.

Watter, S., \& Logan, G. D. (2006). Parallel response selection in dualtask situations. Perception \& Psychophysics, 68, 254-277.

Yeung, N., Botvinick, M. M., \& Cohen, J. D. (2004). The neural basis of error detection: Conflict monitoring and the error-related negativity. Psychological Review, 111, 931-959. 